\title{
A super-efficient and biosafe hemostatic cotton gauze with controlled balance of hydrophilicity/hydrophobicity and tissue adhesiveness
}

Huaying He

Fujian Normal University

Weikang Zhou

Fujian Normal University

Jing Gao

Fujian Normal University

Fan Wang

Fujian Normal University

Shaobing Wang

Fujian Normal University

Yan Fang

Fujian Normal University

Yang Gao

Fujian Normal University

Wei Chen

Fujian Normal University

Wen Zhang

Fujian Normal University

\section{Yunxiang Weng}

Fujian Normal University

\section{Zhengchao Wang}

Fujian Normal University https://orcid.org/0000-0001-7439-9630

Haiqing Liu ( $\nabla$ haiqingliu@fjnu.edu.cn )

Fujian Normal University https://orcid.org/0000-0003-4457-6848

\section{Article}

Keywords: cotton gauze, hemostatic material, hemostatic gauzes

Posted Date: September 14th, 2021 
DOl: https://doi.org/10.21203/rs.3.rs-903862/v1

License: (c) (1) This work is licensed under a Creative Commons Attribution 4.0 International License. Read Full License 


\title{
A super-efficient and biosafe hemostatic cotton gauze with controlled balance of
} hydrophilicity/hydrophobicity and tissue adhesiveness

Huaying He ${ }^{1 \#}$, Weikang Zhou ${ }^{1 \# \xi}$, Jing Gao ${ }^{1}$, Fan Wang ${ }^{2}$, Shaobing Wang ${ }^{2}$, Yan Fang ${ }^{1 *}$, Yang Gao ${ }^{1}$, Wei Chen ${ }^{1 *}$, Wen Zhang ${ }^{1}$, Yunxiang Weng ${ }^{1}$, Zhengchao Wang ${ }^{2}$, Haiqing Liu $^{1,3^{*}}$

${ }^{1}$ College of Chemistry and Materials Science, Fujian Provincial Key Laboratory of Polymer Materials, Fujian Normal University, Fujian 350007, China

${ }^{2}$ Provincial University Key Laboratory of Sport and Health Science, College of Life Sciences, Fujian Normal University, Fuzhou 350007, China.

${ }^{3}$ Engineering Research Center of Industrial Biocatalysis, Fujian Province Higher Education Institutes, Fujian 350007, China

\#These authors made equal contribution

${ }^{\xi}$ Current address: Zijin Middle School, Shanghang 364214, China

${ }^{*}$ Correspondence to: Haiqing Liu (E-mail: haiqingliu@fjnu.edu.cn);

Yan Fang (E-mail: fangyan_YWJ@163.com); Wei Chen (Email: chenwei@fjnu.edu.cn)

\begin{abstract}
Cotton gauze is a widely used topical hemostatic material for bleeding control in military and civil accidents and surgical operations, but its high blood absorption capacity tends to cause extra blood loss of the wounded person, which may increase the risk of shock and death. Therefore, development of rapid hemostatic cotton gauze with less blood loss is of great significance. Herein, we prepared a super-efficient hemostatic cotton gauze whose surface was slightly modified with a catechol compound which features a flexible long hydrophobic alkyl chain terminated with a catechol group. Its hemostatic performance in rat and pig injury models was far superior to standard cotton gauze and Combat Gauze ${ }^{\mathrm{TM}}$. The latter is a well-known commercial gauze for
\end{abstract}


controlling massive hemorrhaging. Additionally, after stoppage of bleeding, the wound sites hardly re-bleed upon the gauze was peeled off. Histological analysis proved that the novel cotton gauze well kept the biosafety of cotton gauze. Interestingly, a similar impressive hemostatic performance was also achieved for chitosan nonwoven gauze modified with the same procedure. Density functional theory calculation and instrumental measurements demonstrate that their extraordinary hemostatic capability is attributable to the highly efficient formation of big and thick primary blood clot made of massive aggregated erythrocytes, due to gauze's effective controlling of blood movement through its blocking effect from tissue adhesion by catechol, platelet activation by cotton fiber, blood absorption by cotton, and hydrophobic effect from long alkyl chain. The methodology and hemostatic mechanisms presented in this work may open a new avenue for developing highly efficient hemostatic gauzes.

\section{INTRODUCTION}

Massive bleeding from nonfatal traumatic wounds is one of the leading causes of death and disability in battlefields and civilian accidents, because significant blood loss causes symptoms such as hypothermia, coagulopathy, acidosis, sepsis, and organ failure. ${ }^{1,2}$ More than $50 \%$ of these mortalities is preventable if emergent and efficient hemostatic measures are applied. This inspires the development of advanced hemostatic products and technologies for prehospital bleeding control of the wounded people, to increase survival rate and reduce medical costs..$^{3-6}$

Cotton gauze has a long history as an effective topical hemostatic fabric for compressible and noncompressible wounds, mainly due to its safety, non-allergy, low cost, adaptability, breathability, stability, blood absorbency, and easy applicability ${ }^{7,8}$. It is still the most widely used hemostat for traumatic bleeding control, although lots of 
1 efficient hemostatic agents have been manufactured and clinically applied in recent two

2 decades..$^{9-12}$ The hemostatic mechanism of cotton gauze counts on the activation of 3 platelets upon contacting with cotton fiber, and its quick wicking of blood fluid, leading to resting of blood cells and platelets to form blood clots. However, it's often seen that excessive large volume of blood is absorbed by cotton gauze before bleeding stops, due to its highly hydrophilic nature, porous structure, and capillary action among the weaved fibers. Those extra blood losses may be the last straw that causes morbidity or mortality because the blood volume in the circulating system is critical.

Many endeavors to enhance the hemostatic efficacy (reduction in blood loss and bleeding time) of cotton gauze have been made in the academic and industrial circles. Z-Medica in USA has commercialized a gauze brand-named QuikClot Combat Gauze ${ }^{\circledR}$ (QCG), which is made by binding inorganic mineral kaolin particles onto rayon/polyester nonwoven. Kaolin can activate clotting factor XII to accelerate blood coagulation reactions, leading to fast thrombus formation. This topical hemostat has been clinically adopted in military, emergency care, and hospital for compressible severe hemorrhage. However, its rapid hemostasis efficacy is subsided because of possible loss of kaolin, and the detached kaolin particles may cause risks of unexpected distal thrombus. To increase binding stability of inorganic particles to cotton fiber, mesoporous chabazite zeolite particles were chemically anchored onto fiber surface by an on-site growth route. ${ }^{8}$ Such a composite cotton gauze has a better topical hemostatic efficiency than QCG. In the rabbit lethal femoral artery injury model, blood loss of chabazite zeolite-cotton gauze was only about $40 \%$ less than that of QCG. For these two kinds of hybrid cotton gauzes, it's obvious that they still absorb large precious 
1 volume of blood during bleeding control. Tuning the wettability of a hemostatic fabric

2 was proposed to address this concern. ${ }^{13}$ A Janus gauze consisting of a top hydrophobic

3 fabric layer and a bottom hydrophilic cotton fabric layer was developed. It was thought

4 that the hydrophilic layer absorbed blood to expedite clotting, while the hydrophobic

5 layer gives a pressure to inhibit blood diffusion through gauze in lengthways. However,

6 blood permeation in warp and weft directions of the bottom cotton fabric layer is un-

7 avoidable, resulting in losses of valuable blood yet. Furthermore, in most cases, blood

8 seepage from the seam between the hemostatic fabric/wound surface, which leads to

9 massive blood loss as well. A composite gauze with a superhydrophobic

10 Poly(vinylidene fluoride)/carbon nanofiber (PVDF/CNF) bottom coating layer was

11 reported to have fast hemostatic capability and no re-bleeding potential, because of

12 synergetic effects from CNF's acceleration of fibrin fiber formation and PVDF's

13 repellency of blood. ${ }^{14}$

Therefore, controlling of movement of blood fluid at the gauze/tissue contact

15 surface and in gauze is the key in designing a highly efficient hemostatic gauze. Inspired

by mussel foot protein's good adhesion to wet substrates, ${ }^{15-17}$ we speculate that cotton

17 gauze with catechol group on its surface may help it adhere to blood-wetted tissue, in

18 order to hinder blood seepage from the seam of gauze/tissue contact surface; meanwhile,

19 the strong interaction among cotton fibers through catechol linkage may slow down

20 blood diffusion in gauze. Thus, in view of the great importance of fiber structure,

21 wettability, and wet biological tissue adherence to hemostatic materials, herein, we

22 design and prepare a novel hemostatic cotton fabric, which is made by slightly grafting

23 a catechol compound (Fig. 1a) with a C15 alkyl side chain onto the fabric surface. This 
1 hemostatic fabric integrates wet tissue adhesiveness from catechol group,

2 hydrophobicity from long alkyl chain, absorbency and breathability from cotton fiber,

3 into one hemostatic device. In rat femoral artery and liver laceration, and pig femoral

4 artery injury models, this modified cotton gauze shows very limited blood permeation

5 through the gauze, no blood oozing out from the seam of gauze/wound contact surface,

6 short bleeding time and reduced blood loss. Its biosafety is comparable to cotton gauze,

7 as suggested by the cytocompatibility and histological examinations. This idea and

8 methodology were also successfully applied to chitosan nonwoven fabric to fabricate a

9 gauze with high hemostatic capability.

(a)
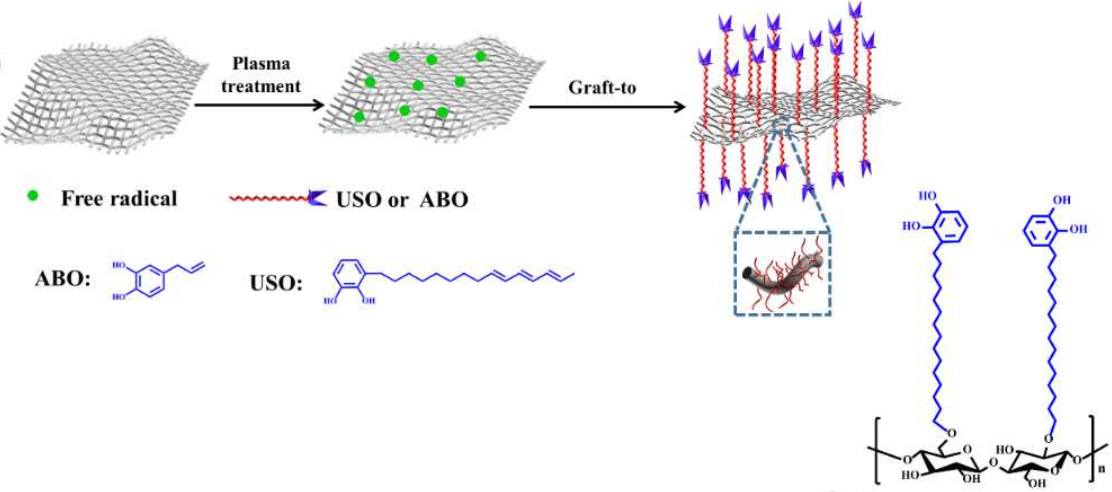

(b)

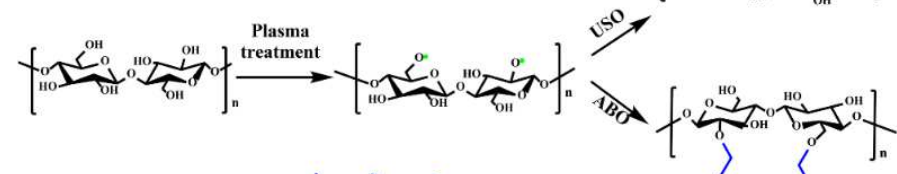

(c)
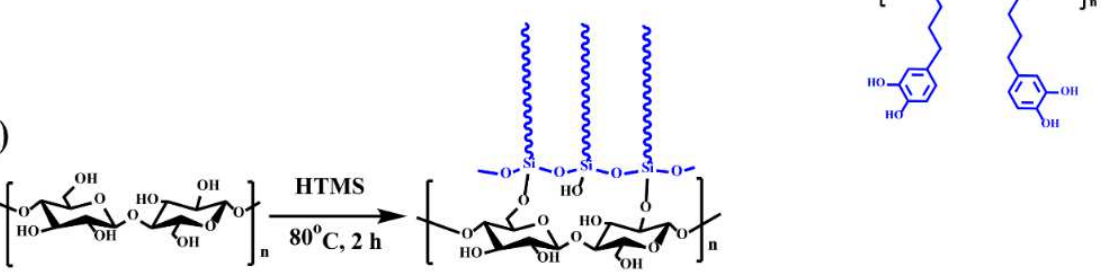

Fig. 1 (a) Reaction scheme for grafting catechols onto cotton gauze. Surface grafting reactions of (b) 4-allyl-1,2-benzenediol (ABO) and 1,2-benzenediol-3-(9,11,13pentadecatrienyl) (USO), and (c) hexadecyltrimethoxysilane (HTMS) onto cotton cellulose macromolecular chain. 


\section{Results}

\section{Chemical composition and surface structure}

Free radicals can be readily generated on cotton gauze by plasma treatment. Through radical initiated reaction with the double bonds in USO, which can be grafted onto cotton gauze surface. Thus, a long alky side chain with a catechol end group was introduced onto cotton fiber (Fig. 1a, b). Its chemical structure is confirmed by its solid state ${ }^{13} \mathrm{C}$ NMR spectrum (Fig. 2a). The peaks at region of 60-70 ppm are attributed to $\mathrm{C} 6$ of cellulose, while the signals at region of 70-80 ppm is assigned to $\mathrm{C} 2, \mathrm{C} 3$, and C5 of cellulose. The peaks at region of 80-95 and 100-110 ppm correspond to C4 and C1, respectively. ${ }^{18}$ Compared with cotton gauze, several new peaks appear for USO-ggauze, i.e., broad peaks at 120-150 ppm corresponding to unsaturated carbons on benzene ring; peaks at region of 10-40 ppm assigning to methyl and methylene groups of the alkyl chain of USO. Therefore, ${ }^{13} \mathrm{C}-\mathrm{NMR}$ spectra indicates that USO is successfully grafted onto cotton gauze surface.

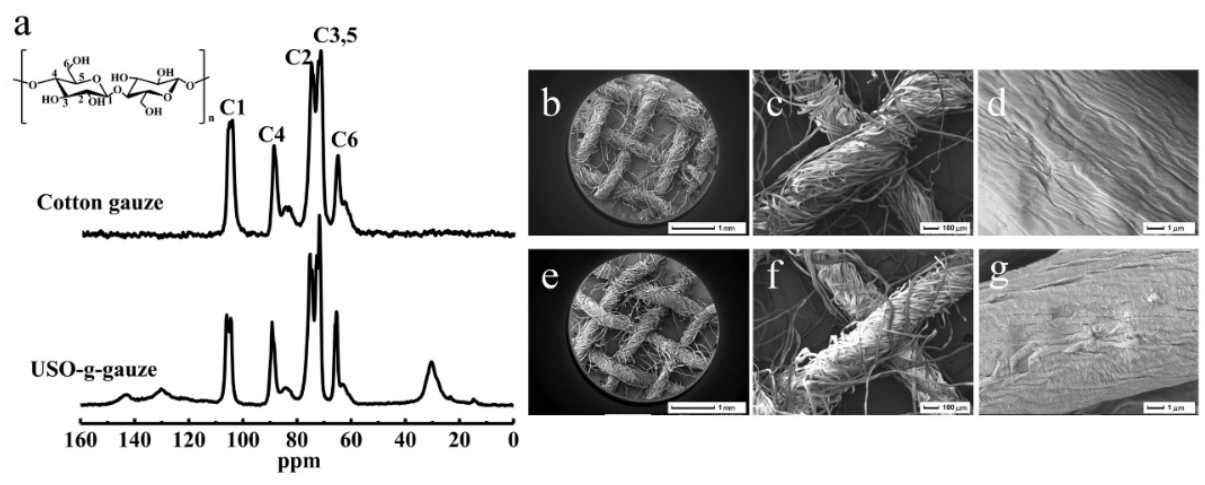

Fig. 2 (a) Solid-state ${ }^{13} \mathrm{C}$ NMR spectra of gauzes. SEM images of (b-d) cotton gauze and (e-g) USO-g-gauze at different magnifications.

FTIR and XPS also prove that ABO, HTMS and USO were successfully grafted onto the surface of cotton gauze (Figs. S2 and S3). For cotton gauze, characteristic 
absorption bands at $3200-3600 \mathrm{~cm}^{-1}\left(v_{\mathrm{O}-\mathrm{H}}\right), 1610 \mathrm{~cm}^{-1}\left(v_{\mathrm{C}-\mathrm{C}}\right), 1435 \mathrm{~cm}^{-1}\left(\delta_{\mathrm{C}-\mathrm{O}-\mathrm{C}}\right), 1125 \mathrm{~cm}^{-}$

${ }^{1}\left(v_{\mathrm{C}-\mathrm{O}-\mathrm{C}}\right)$ are present. In the spectra of ABO-g-gauze, HTMS-g-gauze, and USO-ggauze, a new band at $2846 \mathrm{~cm}^{-1}$ appears for $v_{\mathrm{C}-\mathrm{H}}$ of $-\mathrm{CH}_{2}$ - groups of ABO, HTMS, and USO grafted on the gauze. C1s XPS spectra shows the relatively strongest peak of USO-g-gauze is $\mathrm{C}-\mathrm{C}$ bond, while that of cotton gauze is $\mathrm{C}-\mathrm{O}-/ \mathrm{C}-\mathrm{OH}$ bond (Fig. S3b). This is in accordance with the abundance of C-C bond from the long aliphatic chain grafted on USO-g-gauze.

\section{Surface morphology of gauzes}

Cotton gauze consists of interwoven cotton yarns (Fig. 2b-g). Pores including macropores (among yarns), capillaries (among fibers), meso- and micro-pores (inner fiber) are abundant in the gauze (Fig. 2b). After plasma treatment and radical initiated graft reaction with USO, the interwoven fiber network structure is well maintained, while the surface roughness of cotton fiber slightly increases (Fig. 2g). On the one hand, the improved surface roughness is due to the deepening effect of plasma etching. ${ }^{19} \mathrm{On}$ the other hand, the USO thin layer grafted on the fiber surface may also enhance roughness. ${ }^{20}$ In fact, the well maintenance of fiber/yarn/fabric morphology is also found for ABO-g-gauze and HTMS-g-gauze (Fig. S4).

\section{Wettability of gauzes}

The wettability of a gauze has a large effect on blood fluid absorption, protein adsorption and blood cell adhesion. For instance, it is reported that a material with a water contact angle (WCA) of $40-70^{\circ}$ is suitable for adhesion of various cells. ${ }^{21}$ The wettability of gauze by simulated body fluid (SBF) and fresh rat blood was evaluated by applying $200 \mu \mathrm{L}$ of these liquids onto a gauze. As shown in Fig. 3a, a water droplet 
1 immediately spreads over and diffuses into gauze upon dripping onto gauze surface,

2 due to cotton gauze has a robust intrinsic hydrophilicity and capillary structure. ABO-

3 g-gauze exhibits similar wetting behavior, but the blood spreading area on ABO-g-

4 gauze surface is smaller than that on cotton gauze within a same time period (Fig. 3b).

5 HTMS-g-gauze shows a high hydrophobicity with a contact angle of $132.6^{\circ}$. Water or

6 blood droplet neither spreads radially nor diffuses downwards, but stands on HTMS-g-

7 gauze surface. This is due to this gauze is covered by 16-carbon hydrophobic alkyl

8 chains, whose hydrophobicity prevents blood from diffusing into interior. The instant

9 static WCA of USO-g-gauze is ca. $68.2^{\circ}$. Water or blood exhibits a unique and interesting wetting behavior on USO-g-gauze, namely the droplet gradually and

11 vertically diffuses into gauze in $60 \mathrm{~s}$, while the wetted area on surface is almost identical to the size of the droplet. This is totally different from the easy all-directional liquid pervasion on cotton gauze and ABO-g-gauze.

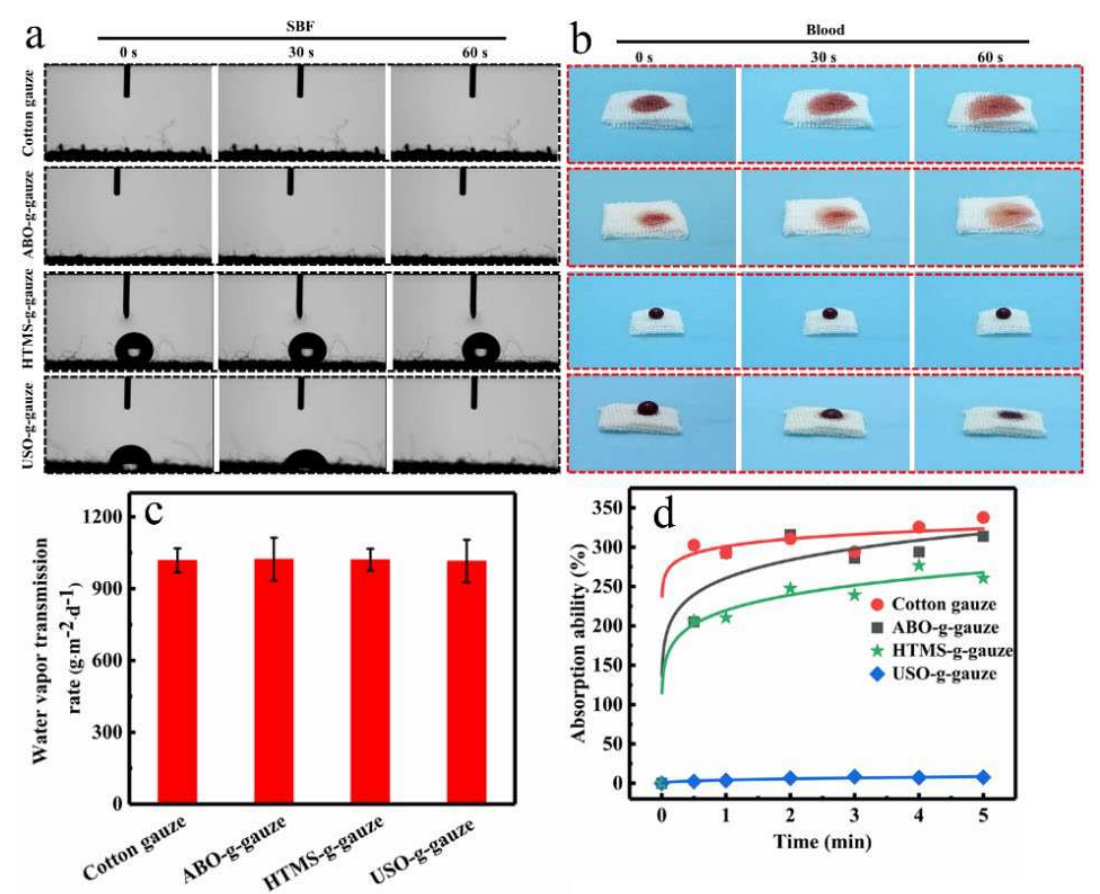

Fig. 3 Wettability of gauzes by (a) SBF and (b) blood. (c) Water vapor permeation rate, and (d) water absorption ratio of gauzes. 


\section{Water vapor permeation rate and water absorption/movement in gauze}

Hemostatic gauze with proper water vapor transmission and liquid absorption can prevent dehydration and excessive accumulation of exudates. Therefore, they can control water/blood loss and create an ideal moist environment for wound healing. ${ }^{22}$ The water vapor permeation rate of cotton gauze, ABO-g-gauze, HTMS-g-gauze, and USO-g-gauze was $1028,1023,1021$, and $1015 \mathrm{~g} \cdot \mathrm{m}^{-2} \cdot \mathrm{day}^{-1}$ at $37{ }^{\circ} \mathrm{C}$, respectively (Fig. $3 c$ ), suggesting that water vapor permeability of the three surface modified gauzes is as good as that of standard cotton gauze, due to their breathable knitted fabric structure.

Cotton gauze and ABO-g-gauze are rapidly wetted by water and sink to the bottom when they are put in water. The hydrophobic HTMS-g-gauze always floats on water surface. However, USO-g-gauze shows a very different behavior, i.e., it initially floats on water surface, but sinks after $30 \mathrm{~min}$ (Suppl. Video 1). USO-g-gauze has a thin USO layer on its surface, weakening its hygroscopicity. This lowers the water absorption rate of USO-g-gauze, which is proven by its water absorption dynamic (Fig. 3d). Therefore, it takes a relatively long period for fully swollen to sink. The water absorption ratio of cotton gauze, ABO-g-gauze, HTMS-g-gauze, and USO-g-gauze is approximately $337.7 \%, 313.9 \%, 7.6 \%$, and $260.5 \%$ at the saturated state (Fig. 3d), respectively. Compared to cotton gauze, the water absorption ratio of USO-g-gauze significantly lose by $77.2 \%$, attributing to the great effect from the very thin hydrophobic USO layer on surface.

The movement of water in gauze was also quantitatively measured by moisture management test (MMT). The variation of wetting time of gauzes (Table 1) is in good correlation to their wettability as shown in Fig. 3a and b. The wetting time (top side) is 
1 as short as $0.19 \mathrm{~s}$ for cotton and ABO-g-cotton gauzes, and substantially increases to

$21.59 \mathrm{~s}$ for USO-g-cotton, and to $15.53 \mathrm{~s}$ for hydrophobic HTMS-g-gauze. As indicated

3 in Table 1, the shorter wetting time is, the faster the water absorption rate and spreading

4 speed is. The water absorption rate of gauzes follows a decreasing order of Cotton

5 gauze $>$ ABO-g-gauze $>$ USO-g-gauze $>$ HTMS-g-gauze, while the water spreading

6 speed is in the order of Cotton gauze $\approx$ ABO-g-gauze $>>$ USO-g-gauze $>>$ HTMS-g-

7 gauze. The changing patterns of these indices suggest that the surface chemical structure

8 of gauze effectively guides moisture movement (wetting, spreading, and diffusion) in

9 gauze, agreeing well with the results shown in Fig. 3. The designed concomitant

10 hydrophobic/hydrophilic structure of USO-g-cotton imparts it with not only proper

11 wetting time and spreading rate, but its ability of water diffusion from one side to the

12 other side (indicated by the cumulative one-way transport capacity, Table 1). Such a

13 unique property would be very helpful for controlling blood movement in gauze and at

14 the gauze/tissue contact surface when it is practically applied as a topical hemostat, as

15 will be shown in the following sections.

16

Table 1 Results of moisture management test of gauzes.

\begin{tabular}{|c|c|c|c|c|c|c|c|}
\hline \multirow[b]{2}{*}{ Sample } & \multicolumn{2}{|c|}{ Wetting time (s) } & \multicolumn{2}{|c|}{$\begin{array}{l}\text { Maximum wetted radius } \\
(\mathrm{mm})\end{array}$} & \multirow{2}{*}{$\begin{array}{c}\text { Water } \\
\text { absorption } \\
\text { rate }(\% / s)\end{array}$} & \multirow{2}{*}{$\begin{array}{c}\begin{array}{c}\text { Spreading } \\
\text { rate }(\mathrm{mm} / \mathrm{s})\end{array} \\
\text { Top side }\end{array}$} & \multirow{2}{*}{$\begin{array}{c}\text { Cumulative } \\
\text { one-way } \\
\text { transport } \\
\text { capacity }\end{array}$} \\
\hline & Top side & $\begin{array}{l}\begin{array}{l}\text { Bottom } \\
\text { side }\end{array} \\
\end{array}$ & Top side & $\begin{array}{l}\text { Bottom } \\
\text { side }\end{array}$ & & & \\
\hline $\begin{array}{l}\text { Cotton } \\
\text { gauze }\end{array}$ & $0.19 \pm 0.00$ & $0.19 \pm 0.00$ & $20.00 \pm 5.00$ & $20.00 \pm 5.00$ & $28.52 \pm 2.86$ & $20.19 \pm 1.40$ & $220.53 \pm 54.12$ \\
\hline $\begin{array}{l}\text { ABO-g- } \\
\text { gauze }\end{array}$ & $0.19 \pm 0.00$ & $0.19 \pm 0.00$ & $10.00 \pm 0.00$ & $10.00 \pm 0.00$ & $11.46 \pm 5.20$ & $18.03 \pm 0.10$ & $370.38 \pm 21.40$ \\
\hline $\begin{array}{l}\text { HTMS- } \\
\text { g-gauze }\end{array}$ & $15.53 \pm 0.58$ & $48.65 \pm 5.65$ & $5.00 \pm 0.00$ & $3.33 \pm 0.36$ & $3.06 \pm 0.17$ & $0.320 \pm 0.01$ & $843.65 \pm 56.37$ \\
\hline $\begin{array}{l}\text { USO-g- } \\
\text { gauze }\end{array}$ & $1.59 \pm 0.47$ & $3.00 \pm 0.00$ & $10.00 \pm 0.00$ & $5.00 \pm 0.00$ & $9.33 \pm 3.50$ & $3.42 \pm 0.64$ & $672.32 \pm 68.44$ \\
\hline
\end{tabular}

17

18

19 
Aggregated erythrocytes and platelets are essential components of blood clot for bleeding control. ${ }^{23}$ SEM was used to explore the interaction of erythrocyte/platelet with gauzes. As shown in Fig. 4, erythrocytes adhere on gauze fiber surface to form aggregates. The unique double concave disk structure of erythrocyte is well maintained,

6 indicating that gauzes don't affect the normal physiological state of erythrocytes.

7 However, the amounts of erythrocyte adhering on gauze fiber surface depend on its wettability. Due to the hydrophobicity of HTMS-g-gauze, on it significantly fewer erythrocytes are observed than on the other three gauzes (Fig. 4c). Cotton gauze accumulates erythrocytes simply by blood fluid absorption, leading to adherence of erythrocytes to its surface (Fig. 4a). However, many erythrocytes aggregate on the fiber surface of ABO-g-gauze and USO-g-gauze (Fig. 4b, d). In addition, the formation of larger platelet aggregations can be observed on USO-g-gauze than on cotton gauze (Fig. $4 \mathrm{e}, \mathrm{f})$. It is clearly seen that some platelets with pseudopods are present on USO-ggauze surface, indicating that platelets have been successfully activated upon contact

16 with gauze fiber. ${ }^{24}$ The above phenomena suggest that USO-g-gauze can catch the main components such as erythrocytes and platelets in plasma to accelerate formation of blood clots. 

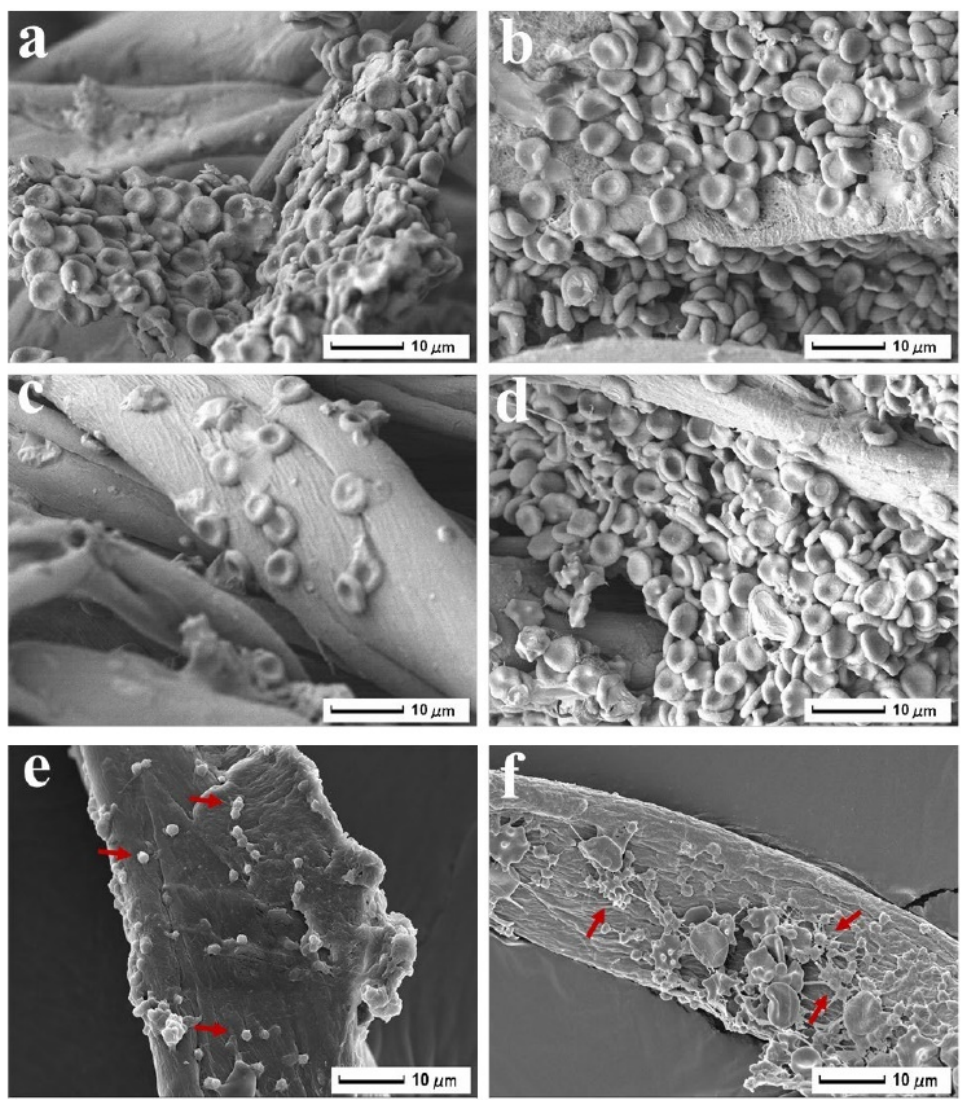

2 Fig. 4 Erythrocytes on (a) Cotton gauze; (b) ABO-g-gauze; (c) HTMS-g-gauze; (d) USO-

3 g-gauze. Platelets on (e) cotton gauze fiber and (f) USO-g-gauze fiber. once again welling out from the wound when gauze is removed after bleeding stops in

\section{Hemostatic performance evaluation}

\section{Bleeding control efficiency in rat femoral artery injury model}

The hemostasis efficacy of gauzes on in vitro trauma was evaluated by using the rat femoral artery injury model. The hemostatic performance of the five gauzes is significantly different from each other (Fig. 5a, Suppl. Video 2). Due to high hydrophilicity, blood quickly permeates into the outmost layer of the stacked four-layer cotton gauze within $1 \mathrm{~s}$. The blood pervasive area on the gauze swiftly expands with increasing time, and the gauze surface is almost fully blood-stained in $60 \mathrm{~s}$. Blood is $5 \mathrm{~min}$. On the unfolded gauze, the four gauze layers are completely wetted by blood 
(Fig. 5-unfolded gauze). These phenomena imply that it absorbs a large volume of blood before bleeding is controlled, and the thrombus is easily destroyed when gauze is removed from the wound (Fig. 5a). The hemostatic performance of ABO-g-gauze is similar to that of cotton gauze, i.e., blood also quickly permeates and wets the whole four-layer ABO-g-gauze. However, the area wetted by blood on ABO-g-gauze is slightly smaller than that on cotton gauze in $60 \mathrm{~s}$, and the red color of the bloody ABOg-gauze is lighter than that of cotton gauze (Fig. 5a). On the unfolded ABO-g-gauze, the piles of the four gauze layers are fully permeated by blood, which is similar to the case of cotton gauze (Fig. 5-unfolded gauze). After ABO-g-gauze is removed posthemostasis, re-bleeding occurs. However, the blood-stained area around wound is obviously less than that in the cotton gauze group (Fig. 5a), suggesting that the hemostatic performance of $\mathrm{ABO}-\mathrm{g}-\mathrm{gauze}$ is improved to some extent.

When the hydrophobic HTMS-g-gauze comes into contact with trauma, blood does not diffuse vertically into the top gauze layer, but it steadily seeps out of the seam of gauze/tissue surface. On the unfolded gauze, only a small area of the first gauze layer in contact with the wound is stained by blood in $60 \mathrm{~s}$ (Fig. 5-unfolded gauze). The gauze is taken away in 10 min when bleeding ceases, it is seen that blood stains the vicinity of the wound and even wet the gauze underneath the thigh, and severe re-bleeding occurs (Fig. 5a), indicating its hemostatic ability is far worse than cotton gauze. For comparison, when HTMS-g-gauze is compressed onto the bleeding wound, blood spills and still seeps out even after 2-minute-compressing (Suppl. Video 3). This is due to the hydrophobic HTMS-g-gauze has a poor moisture management ability which is not 
1 helpful for hemostat. When applied to a bleeding trauma, the hydrophobic HTMS-g-

2 gauze inhibits blood wetting, absorption, wicking and diffusion into the upper gauze

3 layer (Fig. 3 and Table 1), resulting in poor hemostatic performance. As for QCG gauze,

4 an acclaimed gauze for effective controlling of severe bleeding, the outflowing blood

5 diffuses into the four-layer gauze in $1 \mathrm{~s}$, but the blood-stained area on the outmost layer

6 gauze is smaller than that on cotton gauze and ABO-g-gauze. The unfolded gauze,

7 which is removed from the post-hemostatic wound, has blood-stains on all four gauze

8 layers (Fig. 5-unfolded gauze). And the wound re-bleeds rather than maintains

9 hemostatic state (Suppl. Video 2). Very surprisingly, when USO-g-gauze is in contact

10 with the bleeding wound, blood neither diffuses through to the top gauze layer nor seeps

11 out of the seam of gauze/wound surface no matter the gauze is applied with (Suppl.

12 Video 3) or without pressure (Suppl. Video 2). Compared with the other four types of

13 gauze, there is only one small blood-stain on the first two gauze layers of four-layers in

14 total (Fig. 5-unfolded gauze). In the post-hemostasis, it is amazingly found that the

15 wound and its vicinity is dry and clean without obvious blood stain, and no re-bleeding

16 occurs. These exceptional phenomena strongly indicate that the USO-g-gauze has

17 excellent hemostatic efficiency on the rat femoral artery injury. In an extended similar

18 work (Section 5 of SI), we found that chitosan nonwoven whose surface was slightly

19 grafted with USO also showed significantly enhanced hemostatic performance similar

20 to USO-g-gauze, namely controlled blood spreading and diffusion in gauze, no re-

21 bleeding upon gauze removal, and small blood loss, compared to mean performance of

22 pristine chitosan nonwoven gauze (Fig. S5, and Suppl. Video 4). 
2 time of ca. $356 \pm 15 \mathrm{~s}$; The hemostasis time of ABO-g-gauze is ca. $174 \pm 13 \mathrm{~s}$, down

3 from ca. $180 \pm 20 \mathrm{~s}$ of cotton gauze; that of USO-g-gauze astonishingly fells to ca. 34

4 s from 147 s of QCG gauze (Fig. 5b). Compared with QCG gauze, the hemostatic time

5 of USO-g-gauze drops by $77 \%$. Accordingly, the blood loss follows a decreasing order of HTMS-g-gauze $(1.04 \pm 0.09 \mathrm{~g})>$ cotton gauze $(0.52 \pm 0.03 \mathrm{~g}) \approx \mathrm{ABO}$-g-gauze $(0.49$ \pm 0.08 g) $>$ QCG gauze $(0.42 \pm 0.03$ g) $>>$ USO-g-gauze $(0.12 \pm 0.03$ g) (Fig. 5c)

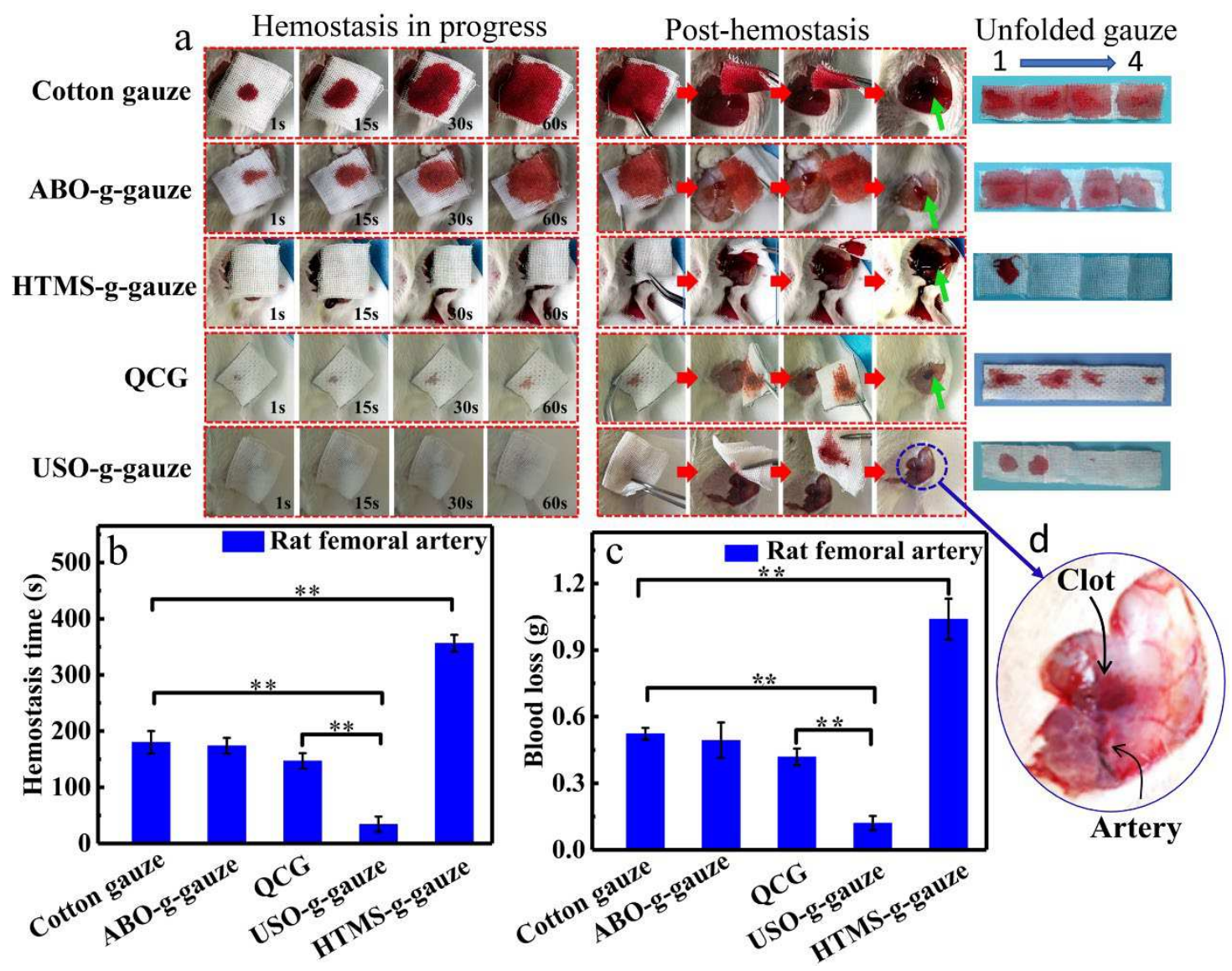

11 Fig. 5 (a) From left to right: gauze was put on the bleeding rat femoral artery injury, 
four gauze layers were unfolded. "1" was the layer directly contacted with the wound, "4" was the outmost layer. (b) Hemostatic time and (c) total blood loss in the rat femoral artery model. (d) Enlarged photo of rat femoral artery injury after hemostasis. This photo was taken right after the gauze was removed.

\section{Hemostasis in rat liver injury model}

The gauze's hemostatic performance in a non-compressible wound is evaluated by using the rat liver laceration model. Their behaviors of blood diffusion, flow at the gauze/tissue contact surface, and re-bleeding are similar to the hemostasis on the rat femoral artery injury model (Fig. 6a and Fig. 6-unfolded gauze, Suppl. Video 5). Obviously, the hemostatic efficacy of USO-g-gauze is significantly better than the other four gauzes. When USO-g-gauze is removed from the wound in post-hemostasis, no fresh blood wells out from the liver wound. The hemostatic time of cotton gauze, ABOg-gauze, HTMS-g-gauze, QCG gauze, and USO-g-gauze is $172 \pm 20 \mathrm{~s}, 153 \pm 15 \mathrm{~s}, 344$ $\pm 19 \mathrm{~s}, 96 \pm 12 \mathrm{~s}$, and $32 \pm 4 \mathrm{~s}$, respectively (Fig. 6b). In addition, the blood loss of cotton gauze, ABO-g-gauze, HTMS-g-gauze, QCG, and USO-g-gauze is $0.39 \pm 0.08 \mathrm{~g}$, $0.37 \pm 0.03 \mathrm{~g}, 0.98 \pm 0.09 \mathrm{~g}, 0.13 \pm 0.07 \mathrm{~g}$, and $0.03 \pm 0.01 \mathrm{~g}$, respectively (Fig. 6c) Compared with QCG gauze, the hemostatic time and blood loss of USO-g-gauze is reduced by $67 \%$ and $77 \%$, respectively. Therefore, the high hemostatic ability of USOg-gauze on the non-compressible liver injury is also confirmed.

In both rat femoral artery injury and liver laceration models, the survival rate within 120 min varies from one gauze to another. It is $100 \%$ for rats treated with USOg-gauze, compared to $20 \%$ for QCG, and to no survival for cotton, ABO-g-gauze and HTMS-g-gauze (Figs. S6a and b). The survival rate is in accordance with the hemostatic 
1 efficacy, i.e., blood loss and hemostasis time of the gauzes as demonstrated in Figs. 5 and 6.
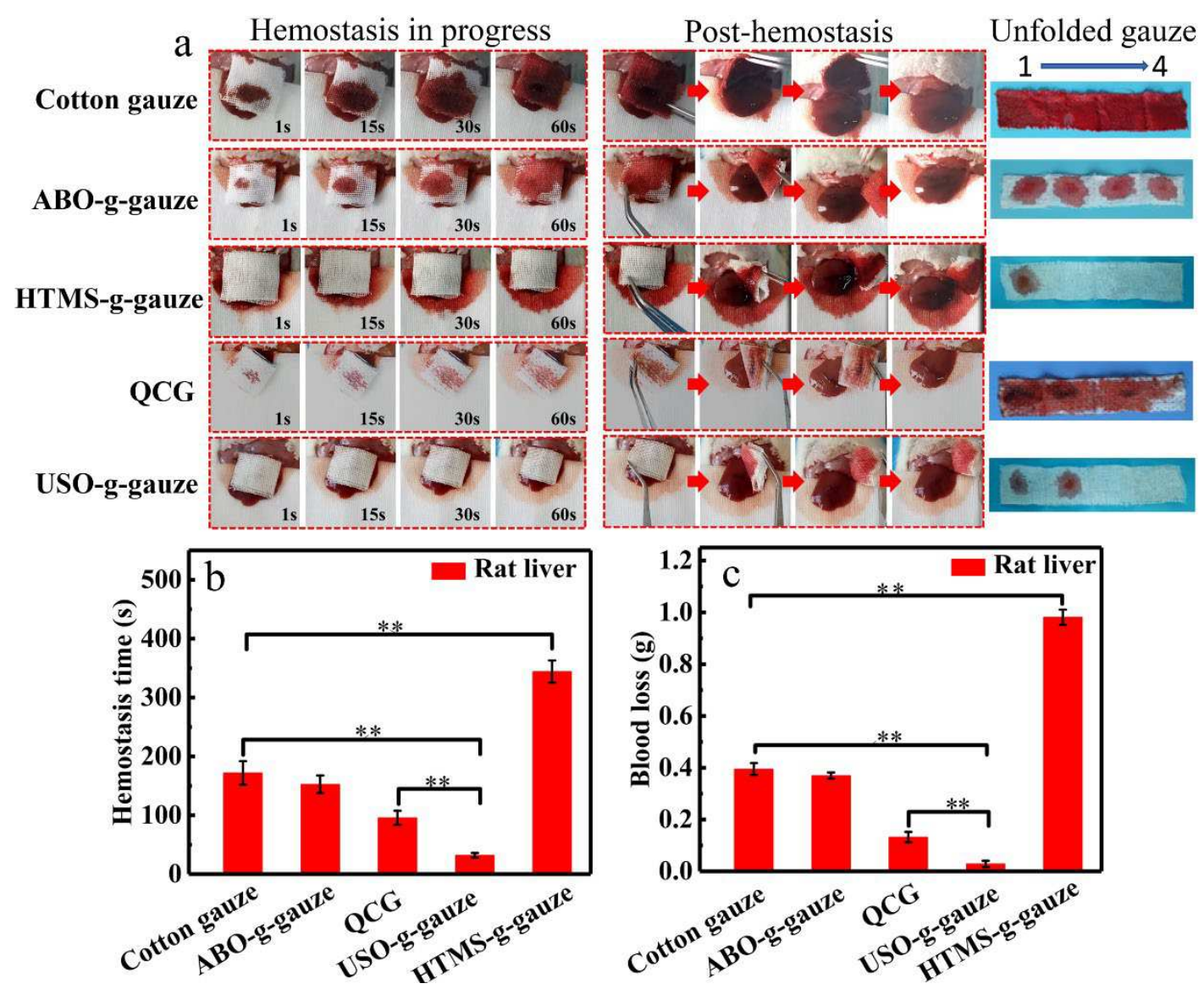

Fig. 6 (a) From left to right: gauze was put on the bleeding rat liver injury, then it was removed from the wound after hemostatic state was reached; The stacked four gauze layers were unfolded. "1" was the layer directly contacted with the wound, "4" was the outmost layer. (b) Hemostatic time and (c) total blood loss of gauzes in the liver injury models.

\section{Hemostatic performance in pig femoral artery injury model}

In order to further evaluate the hemostatic performance of gauzes on massive bleeding wounds, the pig femoral artery injury model is used. The cotton gauze is wrapped around the wound for 3 min, then opened up to check the wound. As shown 
in Fig. 7a and Suppl. Video 6, the outmost cotton gauze layer is immediately wetted by blood upon contact with the trauma. The blood diffusion area steadily increases as time goes by. Fresh blood continues flowing out from the wound when the cotton gauze is removed after 3 min (Fig. 7c). In contrast, USO-g-gauze shows far better hemostatic performance (Suppl. Video 7). Only a small blood-stain is observed on the outmost gauze layer in $60 \mathrm{~s}$ (Fig. 7b). Three min later, re-bleeding does not happen upon the gauze is removed from the wound (Fig. 7d). Because this is a severe bleeding wound, a relatively big area is blood-wetted in the first layer, but the $4^{\text {th }}$ layer is stained by a small blood domain only. This suggests blood diffusion in the vertical and radial directions is largely restricted in USO-g-gauze. It should be pointed out that the accurate hemostatic time of every gauze is not measured on this injury model, because it is hard to judge when the wound stops bleeding due to the wound is wrapped by gauze and rebleeding often occurs upon uncovering wound. Therefore, at the time interval of $3 \mathrm{~min}$, the blood mass absorbed by gauze is measured to roughly reflect the blood loss. This is $0.80 \pm 0.12 \mathrm{~g}$ for USO-g-gauze, while that of cotton gauze, ABO-g-gauze, QCG, and HTMS-g-gauze is $5.12 \pm 0.34 \mathrm{~g}, 4.16 \pm 0.40 \mathrm{~g}, 3.93 \pm 0.30 \mathrm{~g}$, and $8.20 \pm 0.34 \mathrm{~g}$, respectively (Fig. 7e). Therefore, the blood loss from the pig femoral artery wound treated with USO-g-gauze is only $15.6 \%$ and $20.4 \%$ of that with cotton gauze and QCG, respectively. In fact, the hemostasis time of USO-g-gauze on this wound model is less than $3 \mathrm{~min}$, as suggested by the fact that no occurrence of re-bleeding upon uncovering the wound (Fig. 7d).

Hemostasis on the pig skin laceration model is also examined since its structure is very similar to human skin. A regular cut with a length of $2 \mathrm{~cm}$ and a depth of $1 \mathrm{~cm}$ was made with a scalpel, then a four-layer gauze was applied onto the wound. The dynamic hemostatic process of the four gauzes demonstrates that blood diffusion and absorption, 
1 and blood flowing underneath gauze, are very similar to the hemostasis on the rat

2 femoral artery injury and liver laceration models. Blood even rarely stains the

3 hydrophobic HTMS-g-gauze, but oozes out from the seam of gauze/skin surface (Fig.

4 S7). The blood loss of the cuts treated with cotton gauze, ABO-g-gauze, QCG, HTMS-

5 g-gauze, and USO-g-gauze is $0.55 \pm 0.04 \mathrm{~g}, 0.32 \pm 0.03 \mathrm{~g}, 0.22 \pm 0.02 \mathrm{~g}, 0.71 \pm 0.02 \mathrm{~g}$,

6 and $0.032 \pm 0.01 \mathrm{~g}$ (Fig. 7f), respectively. Compared with cotton gauze and QCG, the

7 blood loss for USO-g-gauze reduces by ca. $94 \%$ and $85.5 \%$, respectively. The results

8 of the pig femoral artery injury and skin laceration models further justify that USO-g-

9 gauze has excellent hemostatic efficacy for severe bleeding wounds.
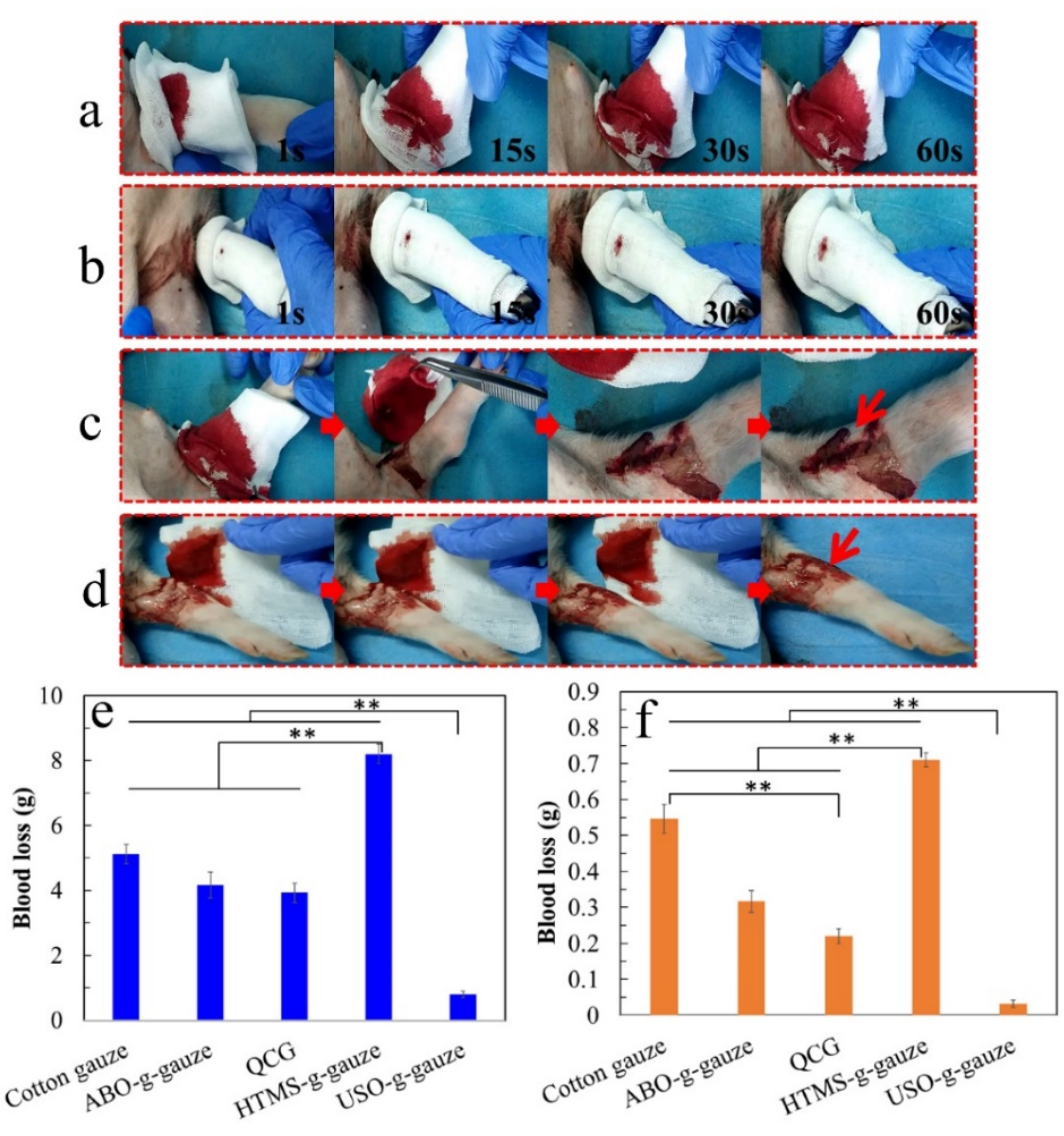

11 Fig. 7 Hemostasis in the pig femoral artery injury models. Hemostasis process of (a) cotton gauze, and (b) USO-g-gauze. The status of wound after (c) cotton gauze and (d) USO-g-gauze were removed after it was treated for $3 \mathrm{~min}$. Blood loss of gauzes in the pig (e) femoral artery and (f) skin injury models. 


\section{Hemostatic mechanism of the highly efficient USO-g-gauze}

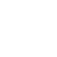

standard cotton gauze. ABO-g-gauze not only concentrates blood components due to its quick blood absorption ability (Fig. 3d), but also catches blood cells by its tissue adhesive catechol groups (Fig. 4b), so the hemostatic efficiency of ABO-g-gauze is slightly improved. The hemostatic performance of HTMS-g-gauze is significantly inferior to that of cotton gauze, because of strong repellence of blood fluid by the highly hydrophobic HTMS alkyl chain (Figs. 3 and 4). However, the USO-g-gauze containing a hydrophobic long alkyl chain with a tissue adhesive catechol end group exhibits impressively excellent hemostatic efficacy.

Why has USO-g-gauze the most exceptional hemostatic capability among those gauzes? To better understand the nature behind this feature of this new hemostatic cotton gauze, we initially carried out the detailed density functional theory (DFT) calculations to investigate the adsorption interaction of sixteen different kinds of amino acid molecules (they are essential components of tissue keratin protein ${ }^{25}$ ) with USO-g-gauze, as illustrated in Fig. S8. More details have been provided in Section 8 of SI. The adsorption energies $\left(\Delta \mathrm{E}_{\mathrm{ads}}\right)$ of these amino acids to USO-g-gauze are calculated by considering the main non-covalent interaction modes including $\pi-\pi$ stacking and hydrogen bond interactions.

Our computed results reveal that the amino acids containing $\pi$-conjugated benzene ring, such as phenylalanine $(\mathrm{F})$ and tyrosine $(\mathrm{Y})$, can be effectively adsorbed on the catechol of USO-g-gauze through synergistic actions of $\pi-\pi$ stacking and hydrogen bonding (Figs. $8 \mathrm{C}$ and $\mathrm{S} 8$ ), where the computed $\Delta \mathrm{E}_{\text {ads }}$ values are as large as 0.621 and $0.729 \mathrm{eV}$, respectively. Comparatively, all the remaining fourteen amino acids without 
$\pi$-conjugated ring can effectively interact with the catechol of USO-g-gauze by adopting double hydrogen bonds, as presented in Fig. 8C and S8. All these hydrogen bonding distances are in the range of $1.69 \sim 1.80 \AA$ (Table $\mathrm{S} 2$ ), and the calculated $\Delta \mathrm{E}_{\text {ads }}$ values are as big as $0.570 \sim 0.639 \mathrm{eV}$ (Fig. S8), indicating strong interaction force between them.

Furthermore, we also examine the effect of relative position between two $\mathrm{OH}$ groups on the benzene ring on $\Delta \mathrm{E}_{\mathrm{ads}}$ of a model amino acid glycine (G) on USO-g-gauze (Fig. S9). The computed results reveal that when the relative position between the two $\mathrm{OH}$ groups is changed from the original ortho- to meta- to para- arrangements, $\Delta \mathrm{E}_{\text {ads }}$ values for $\mathrm{G}$ is reduced from 0.617 to $0.502 / 0.419$ and then to $0.397 / 0.384 \mathrm{eV}$. Further, when the two $\mathrm{OH}$ groups are even separated by three $\mathrm{H}$ atoms, small $\Delta \mathrm{E}_{\text {ads }}$ (ca. $0.383 / 0.355 \mathrm{eV}$ ) is attained. Clearly, with increasing the spacing distance between the two $\mathrm{OH}$ groups, $\Delta \mathrm{E}_{\text {ads }}$ for the model amino acid decreases significantly, in view of the fact that double hydrogen bonds cannot be effectively formed or only a single hydrogen bond can be formed (Fig. S9). Therefore, the relative position between two $\mathrm{OH}$ groups on the benzene ring has an important influence on $\Delta \mathrm{E}_{\mathrm{ads}}$, where the ortho position can bring the largest $\Delta \mathrm{E}_{\mathrm{ads}}$ due to formation of two hydrogen bonds. Further, when removing either of the two $\mathrm{OH}$ groups in catechol of USO-g-gauze (Fig. S10), the computed $\Delta \mathrm{E}_{\text {ads }}$ values $(0.248$ and $0.355 \mathrm{eV})$ is about half of that of the corresponding structure with two hydrogen bonds, which means that both hydrogen bonds can be effectively formed simultaneously between the relevant amino acids and catechol in USO-g-gauze. Obviously, all of these can reflect the superior structural match to form double H-bonds between the amino acids and the catechol of USO.

Overall, USO-g-gauze with a long alkyl chain terminated with a catechol group can effectively interact with all these amino acids via double hydrogen bonds or the synergistic action of $\pi-\pi$ stacking and hydrogen bonding. These non-covalent 
interactions contribute to USO-g-gauze's strong tissue adhesiveness, where the catechol at the end of alkyl chain can play a crucial role.

Apart from the above molecular level analyses of adhesion interaction, the adhesion force (or peeling force) of those gauzes on fresh wet rat femoral tissue is measured and shows obvious variation from one to another. As expected, the hydrophobic HTMS-g-gauze has the lowest peeling force of $24 \mathrm{mN}$, while USO-ggauze shows the largest force of $90 \mathrm{mN}$, which is ca. two times as much as that of cotton gauze (Fig. S11). From Suppl. Video 3, the adhesion of ABO-g-gauze and USO-gchitosan to wound tissue is obviously perceivable when they were peeled off from the wounds, while it is less noticeable in the cases of cotton gauze etc (Suppl. Video 2). This vividly confirms the existence of adhesive interaction between catechol and tissue, but such non-covalent adhesion can be broken with mild peeling forces. In the case of the double $-\mathrm{OH}$ groups of catechol were modified such as with chelation with $\mathrm{Fe}^{3+}$ or oxidation into quinone, the tissue adhesion force sharply decreases to values close to that of HTMS-g-gauze (Fig. S11). This further confirms that catechol group plays an essential role in the wet tissue adhesion. ${ }^{26-28}$

In order to demonstrate the importance of tissue adhesion of catechol to high hemostatic efficiency, the catechol group on USO-g-gauze is transformed to lower its tissue adhesiveness. Hence, USOFe-g-gauze and USOQu-g-gauze were fabricated. On USOFe-g-gauze, catechol groups readily react with $\mathrm{Fe}^{3+}$ to form a complex, while on USOQu-g-gauze the catechol groups are oxidized into quinone (Fig. S12). Both catechol- $\mathrm{Fe}^{3+}$ and quinone groups have no or weak adhesion to wet tissue. ${ }^{29}$ When USOFe-g-gauze is applied onto the rat femoral artery injury, blood spills (1s 
1 in Fig. 15a, pointed by yellow arrow) and seeps out of the seam of gauze/wound surface

2 (15s in Fig. 15a, pointed by yellow arrow), it also diffuses throughout the whole four

3 gauze layers in $15 \mathrm{~s}$, but thereafter the blood-stained area on the top layer does not

4 expand much, instead blood continuously oozes out from the gauze/tissue contact surface (Fig. S13a). The gauze is removed 5 min later when the wound fully stops bleeding, re-bleeding is observed and much red fresh blood is around the wound (Fig. S13a). Compared to USO-g-gauze, the hemostasis time and blood loss on this wound treated by USOFe-g-gauze significantly increases to $289 \pm 5 \mathrm{~s}$, and $1.32 \pm 0.11 \mathrm{~g}$, respectively (Fig. S13b, c). The instant static WCA of USOFe-g-gauze is $119^{\circ}$ (higher than $68^{\circ}$ of USO-g-gauze), but slowly reduces to $0^{\circ}$ within $60 \mathrm{~s}$ with diffusion of water droplet (Fig. S12b), similar to that occurred for USO-g-gauze. Therefore, the weak adhesiveness of catechol- $\mathrm{Fe}^{3+}$ groups to wet skin tissue, would be responsible for the substantially longer hemostatic time and more blood loss than USO-g-gauze and cotton gauze. Similar phenomena (blood diffusion, seepage, and re-bleeding) are found for the USOQu-g-gauze on the rat femoral artery injury (Fig. S13a). This is also due to the less tissue adhesiveness of quinone groups existing on the USOQu-g-gauze surface. The hemostasis time and blood loss by USOQu-g-gauze are $193 \pm 5 \mathrm{~s}$ and $0.87 \pm 0.21 \mathrm{~g}$, respectively (Fig. S13b, c).

The hemostatic performance of USOFe-g-gauze and USOQu-g-gauze on the rat liver laceration model is similar to that on the rat femoral artery injury model (Fig. S14a). Blood flows down the liver and wets the gauze (underneath the rat liver), which does not occur when USO-g-gauze is applied. On this injury, the hemostatic time and 
1 blood loss by USOFe-g-gauze are $101 \pm 4 \mathrm{~s}$ and $0.48 \pm 0.13 \mathrm{~g}$, respectively; and by

2 USOQu-g-gauze are $65 \pm 4 \mathrm{~s}$ and $0.28 \pm 0.10 \mathrm{~g}$, respectively (Fig. S14b, c). Thus, the 3 catechol group does play a crucial role in controlling traumatic bleeding. The far 4 superior hemostatic potential of USO-g-gauze to $\mathrm{ABO}-\mathrm{g}$-gauze ( $\mathrm{ABO}$ has a three carbons alkyl chain) strongly suggests that a long hydrophobic alkyl chain is also of

6 great importance. Therefore, USO, a catechol compound with a side alkyl chain 7 having 15 carbons is a good candidate compound for surface modification of fabric gauze (such as cotton gauze and chitosan nonwoven) to prepare novel highly efficient hemostatic gauzes.

Certainly, other properties of USO-g-gauze such as its moisture management ability (water absorption, wettability, diffusion, and one-way transportation) are also essential to the high hemostatic efficiency. The blood fluid movement in gauze and around the gauze/tissue surface governed by the unique wetting property and tissue/cell adhesiveness facilitates aggregation of astonishingly massive erythrocytes, as shown in Fig. 8B. There are so many congested erythrocytes that they even fill the quadrilateral macro-pores among warp and weft yarns of the first two gauze layers (the layer directly contacts with tissue is the first layer), but none in the $3^{\text {rd }}$ layer, which are consistent with the observation shown in Figs. 5 and 6-unfloded gauze. The thickness of the erythrocyte layer accumulated on the $1^{\text {st }}$ layer reaches as high as $220 \mu \mathrm{m}$. As wellknown, erythrocytes are the key component of the primary blood clot. Thus, more erythrocytes are aggregated, bigger clot is formed, shorter bleeding time and less blood loss are attained. Therefore, the thick erythrocyte layers in the first two USO-g-gauze 
1 layers serve as clots for effectively controlling bleeding. However, the erythrocyte

2 accumulation ability of cotton gauze is poor as suggested by its sparse distribution on

3 cotton gauze yarns with none in the quadrilateral macro-pores of the whole four gauze

4 layers (Fig. 8A). This is because erythrocytes move along with the fast blood wicking

5 to everywhere in the cotton gauze patch, rather than group together to form a big

6 erythrocyte plug. But such a movement is retarded in USO-g-gauze by the anchoring

7 hydrophobic chain barriers at the interface of USO-g-gauze/tissue and among fibers and 8 yarns.

d. When USO-g-gauze is applied onto a bleeding wound, catechol groups of USO

11 quickly anchor to skin tissue through non-covalent bonds such as hydrogen bond and $\pi-\pi$ stacking, to form dam-like barriers around wound (Fig. 8C). This can hinder and eventually prohibit blood from seepage at the gauze/tissue contact surface. The

14 repelling pressure from the massive hydrophobic interaction among the long alky chains retards blood diffusion into the upper gauze layers, so only the first and second layers are blood-wetted as shown in the rat and liver injury models (Figs. 5 and 6unfolded gauze). Even in the pig injury models, blood has a difficulty in diffusing radially, and only little volume of blood reaches the outmost layer to result in a small blood stain (Fig. 7b). This should contribute to the massive dam-like barriers formed among yarns and fibers due to the interaction between USO catechol and cotton cellulose, hence big blood stream moves in the pores between warp and weft yarns, with small blood streams in other pathways (Fig. 8D). Finally, the blood wicking 
1 capability of the moderately hydrophilic USO-g-gauze fibers facilitate platelets

2 adhesion and activation, and erythrocyte accumulation (Fig. 8D), therefore promoting

3 the formation of blood clot. Thus, the synergistic effects of tissue adhesion,

4 hydrophobic interaction, and hydrophilic fiber structure make USO-g-gauze an

5 excellent hemostatic gauze. The other impressive feature of USO-g-gauze is that no re-

6 bleeding occurs upon removing it from wound after hemostasis, while re-bleeding is

7 often experienced when cotton gauze is used. In the case of cotton gauze, with sparse

8 aggregation of erythrocytes on yarns, it is an essential part of the blood plug (Fig. 8A).

$9 \quad$ The plug would be easily broken since erythrocytes are removed along with peeling-

10 off cotton gauze, leading to secondary bleeding. From Fig. 8B, it's seen that the

11 erythrocytes accumulated at the injury site is so enormous and thick that removal of

12 USO-g-gauze would take away part of erythrocytes, but some erythrocytes (Fig. 5d)

13 remain on site to avoid re-bleeding. The mechanism for no re-bleeding of USO-g-gauze

14 is different from that of Bandage ${ }^{\circledR}$, which is a well-known no re-bleeding hemostatic

15 fabric strip for small bleeding wounds. Bandage's anti-adhesion to tissue relies on the

16 hydrophobic membrane covering on the water-absorbent fabric layer (Fig. S15). The

17 poor adhesion of hydrophobic membrane to fibrin prevents bandage from being a part

18 of blood clot. The no re-bleeding is actually very important when wounded person are

19 relocated or injured tissue/organs are moved accidently, so new blood loss can be

20 substantially avoided. Since the hemostasis of USO-g-gauze is a physical blocking

21 effect rather than change of the body's normal physiologic clotting mechanisms, it

22 would also show hemostatic efficacy for patients with coagulopathy. 


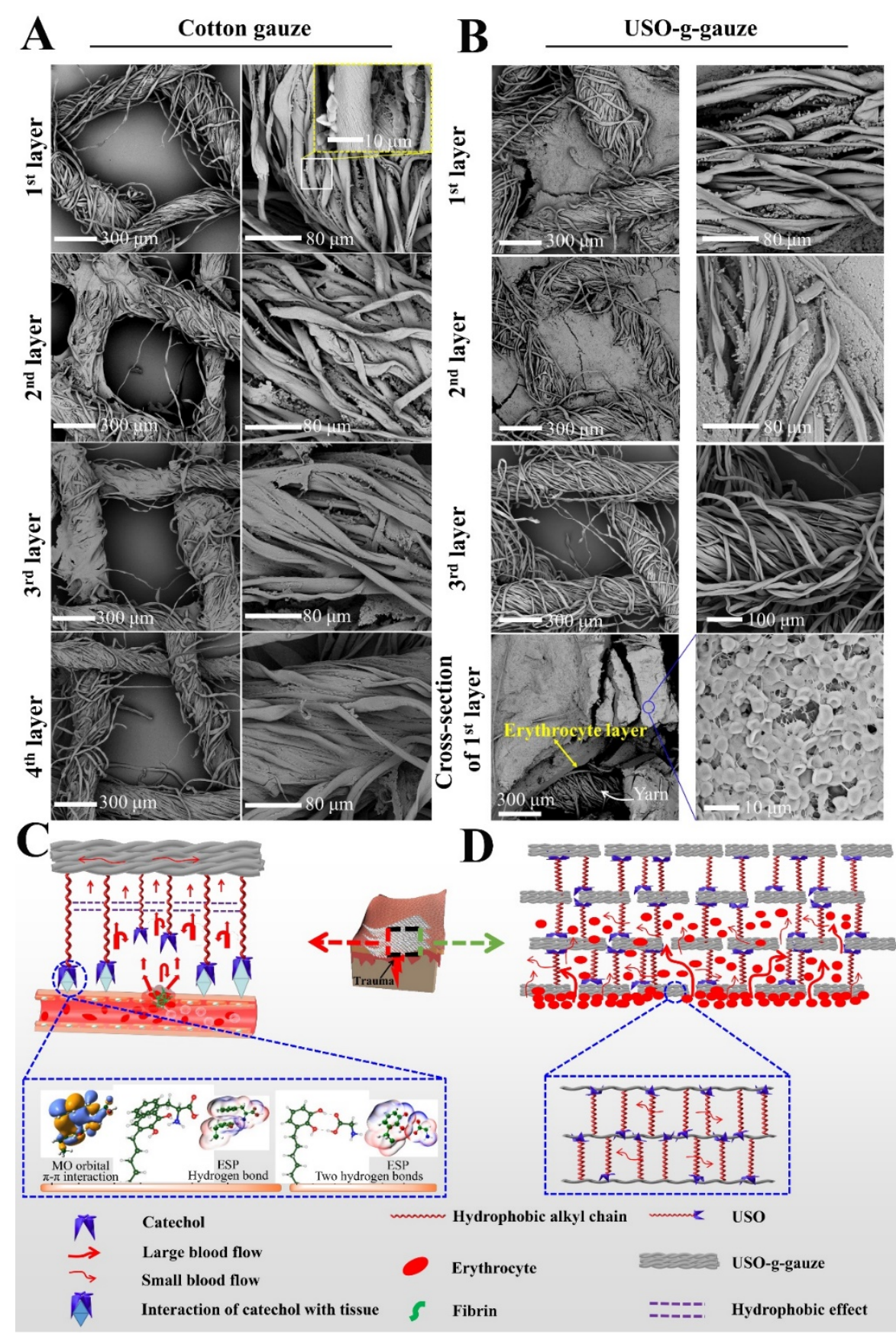

Fig. 8 Aggregation of erythrocytes on cotton gauze and USO-g-gauze after hemostasis

3 in the rate femoral artery injury model; and hemostatic mechanism diagram of USO-g-

4 gauze. (a) On the cotton gauze patch composed of four stacked gauze layers,

5 erythrocytes sparsely distribute on cotton yarns of all layers. The macro-pores among

6 yarns are vacant. (b) On the USO-g-gauze patch composed of four stacked gauze layers,

7 erythrocytes fill the macro-pores among yarns in the first two layers, but are absent in 
1 the pores and on the yarns of the $3^{\text {rd }}$ layer. Cross-section of the $1^{\text {st }}$ layer shows a thick

2 erythrocyte layer. (c) Through adhesive bonds like $\pi-\pi$ stacking interaction and

3 hydrogen-bond between USO's catechol group and wound tissue's amino acid units,

4 dam-like barriers form surrounding the wound. They retard blood seeping out of the

5 tissue surface. The repelling pressure from the hydrophobic effect among long alkyl

6 chains slows down blood wicking movement. (d) Dam-like barriers forming by USO

7 between gauze layers and between fibers, retard blood diffusion in the vertical and

8 horizontal directions, largely confine blood movement in the pores between warp and

9 weft yarns, resulting in large accumulation of erythrocytes.

\section{Biocompatibility of USO-g-gauze}

The growth and proliferation of L929 fibroblasts on USO-g-gauze are shown in

Fig. 9. On the gauze yarn, many green-dyed living fibroblasts with several red-dyed dead cells are observed. Obviously, the fibroblasts proliferate very well with incubation

14 time increasing from 1 to $3 \mathrm{~d}$. The good cell biocompatibility of USO-g-gauze is comparable to that of cotton gauze and ABO-g-gauze (Fig. S16a and b). The fibroblasts can also dwell and grow on HTMS-g-gauze, but the proliferation rate on this hydrophobic gauze is significantly smaller than that on the other three hydrophilic gauzes (Fig. S16c). Therefore, USO-g-gauze can favor cell adhesion and proliferation, due to good balance of hydrophilicity/hydrophobicity and presence of catechol groups on this gauze. 

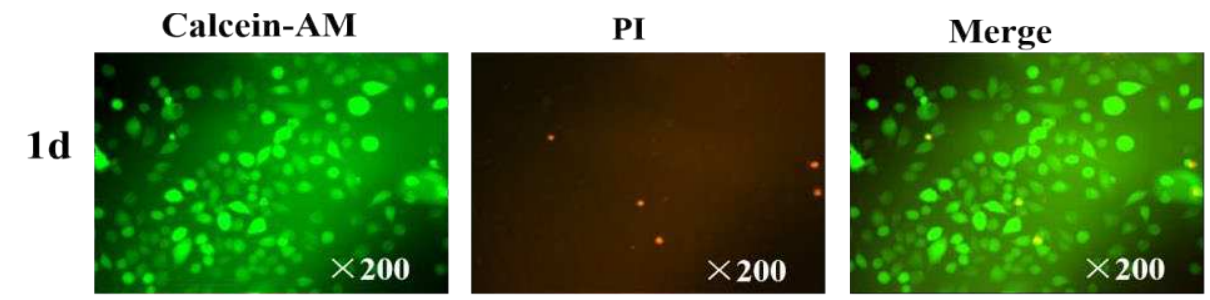

Fig. 10 shows histological changes of the subcutaneous muscle tissue treated with cotton gauze and USO-g-gauze at specific time points (3, 7, 14, 21 days) using H\&E staining and toluidine blue staining. In the cotton gauze-treated tissue, plenty of neutrophils arose around the gauze on the third day after implantation (Fig.10a) and the corresponding number were counted to be about $50 \pm 5$ (Fig. 10c). However, very few neutrophils were observed 7 days later, which reduced to about $2 \pm 1$ after 21 days. Meanwhile, USO-g-gauze had a similar inflammatory response. The density of neutrophils increased in the USO-g-gauze treated tissues since implantation, but rapidly decreased to $4 \pm 1$ after 7 days. In addition to neutrophils, mast cell was another critical effector of inflammation. As shown in the toluidine blue staining (Fig. 10b and d), several mast cells were observed in the tissue section contacting with the cotton gauze and the USO-g-gauze on the seventh days after implantation, but sharply reduced 14 days later within the cotton and the USO-g-gauze treated tissue. The subcutaneous implantation examinations reveal that the surface 
1 modification with USO did not compromise the biocompatibility of cotton gauze, and

2 caused no significant inflammatory responses.

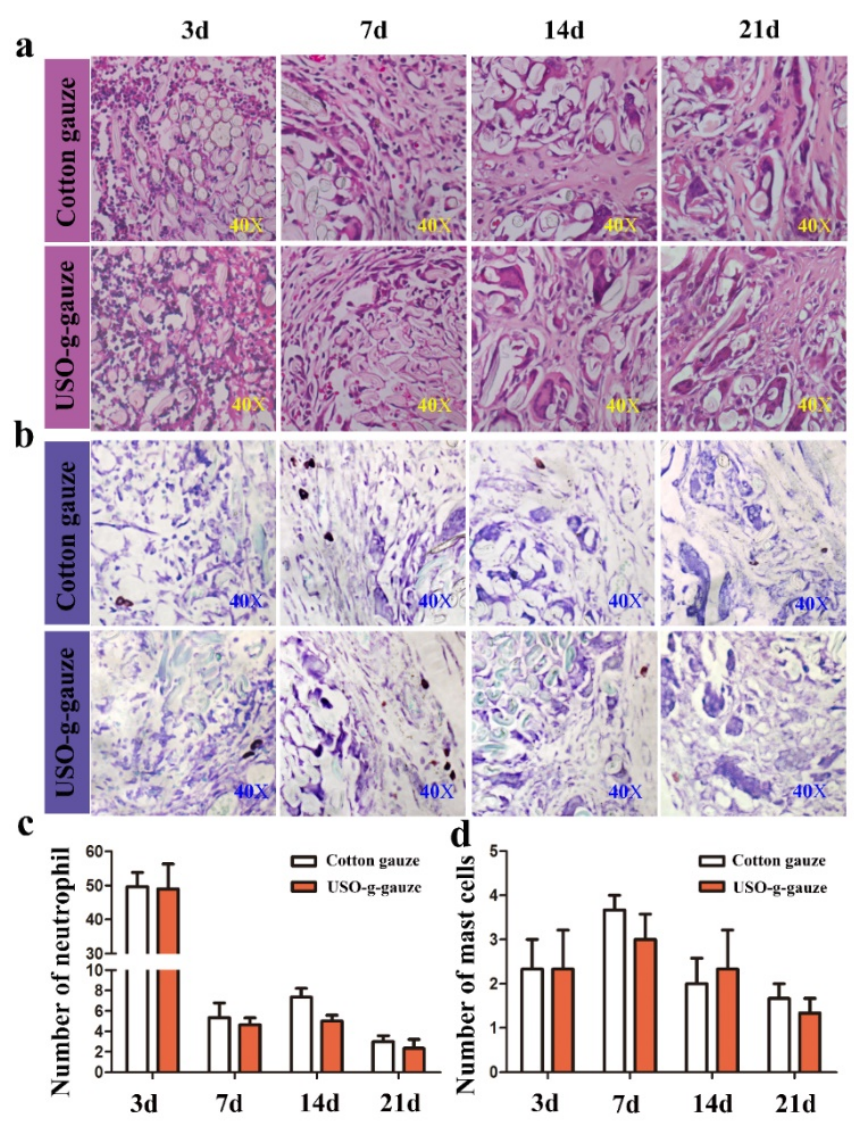

3

7 intervals.

\section{Conclusions}

A novel highly efficient hemostatic cotton gauze was developed through simply grafting a catechol compound USO (with a long hydrophobic alkenyl side chain) onto cotton gauze surface. The special pendent groups offer wet tissue adhesiveness and hydrophobicity to the gauze. On basis of findings of the density functional theory calculation and measurements, a new physical hemostatic mechanism is proposed for this gauze, namely many dam-like barriers are formed at the interface of gauze/wound 
1 tissue surface and among adjacent fibers. These barriers are capable of retarding blood

2 flowing and controlling the movement of bloodstream at the seam of gauze/wound

3 contact surface and in the gauze, resulting in quick aggregation of erythrocyte to form

4 a thick blood clot stopping bleeding. Thus, this gauze can overcome the intrinsic high

5 blood absorption capacity of conventional cotton gauze, making it to significantly

6 reduce additional blood loss from bleeding traumas, and therefore increasing survival

7 rate and decreasing medical such as transfusional costs. Excellent hemostatic efficacy

8 (including short hemostatic time, low blood loss, and no re-bleeding) of this gauze was

9 observed on the rat femoral artery and liver laceration models, pig skin laceration and

10 femoral artery massive bleeding wound. Its hemostatic performance is much superior

11 to standard cotton gauze and QCG. The USO-g-gauze displays similar cell and tissue

12 compatibility to cotton gauze. Such an idea and methodology were also successfully

13 applied to make USO-g-chitosan gauze, whose hemostatic efficacy is much better than

14 the chitosan control. We speculate this magic gauze may find very promising emergency-care and clinical applications for controlling traumatic massive bleeding for wounded soldiers in battlefields, civilians in accidents, patients in operation rooms, and

17 patients with coagulopathy.

\section{Methods}

\section{Materials}

Sterile cotton gauze and chitosan nonwoven fabric were purchased from the commercial market in China. Sodium alginate (SA), acetic acid and anhydrous ethanol were bought from Sinopharm Group Chemical Reagent Co., Ltd. QuikClot Combat Gauze (QCG) was provided by Z-Medica and used as received. The catechol compound 1,2-benzenediol-3-(9,11,13-pentadecatrienyl) (USO) was extracted from the raw 
lacquer provided by Xi'an Raw Paint Research Institute by a reported procedure. ${ }^{30}$ Briefly, $1 \mathrm{~kg}$ of Chinese lacquer sap was dissolved in $2 \mathrm{~L}$ of ethanol under mechanically stirring for $24 \mathrm{~h}$ at room temperature, followed by filtration to collect filtrate. From it USO was obtained after ethanol was removed by vacuum-rotary evaporation at $60{ }^{\circ} \mathrm{C}$. Then the C15 triene urushiol was purified by the gel permeation chromatography (GPC). ${ }^{31}$ Eugenol, triethylsilane (TES), tris(pentafluorophenyl)borane (TPFPB), 3(4,5-dimethyl-2-thiazolyl)-2,5-diphenyl-2-H-tetrazolium bromide (MTT) and hexadecyltrimethoxysilane (HTMS) were purchased from Aladdin Reagent Co., Ltd. All chemicals were used as received without further purification. Sprague-Dawley (SD) rats with weight of ca. 200-250 g were bought from Shanghai Slack Experimental Animal Co., Ltd, China.

\section{Preparation of USO grafted cotton gauze (USO-g-gauze)}

The preparation scheme and chemical reaction of USO-g-gauze are shown in Fig. 1. Cotton gauze was sequentially washed with distilled water and ethanol, two times in each solvent and 30 min each. Then it was dried under nitrogen stream. To introduce free radicals onto gauze, it was treated by a low temperature plasma in $\mathrm{N}_{2}$ at $400 \mathrm{~Pa}, 80$ W for 3 min (PT-5S, Sanhe Poda Co., Ltd, China). The preparation conditions for USO-g-gauze were optimized as described in (Table S1, Section 1 of SI). In an ideal condition, USO-g-gauze was fabricated by placing the plasma-treated gauze in a mixture of $2.0 \mathrm{wt} \% \mathrm{USO} / \mathrm{ethanol}$ and refluxing at $70{ }^{\circ} \mathrm{C}$ for $2 \mathrm{~h}$. Then, after washed 3 times with ethanol, it was dried in a vacuum oven at $80{ }^{\circ} \mathrm{C}$ for $2 \mathrm{~h}$ to obtain USO-ggauze. About $0.1 \mathrm{wt} \%$ USO was grafted, as determined by the gravimetrical method. 4-allyl-1,2-benzenediol (ABO) containing a catechol group was prepared according to the scheme shown in Scheme S1. ${ }^{32}$ Typically, eugenol ( $\left.8.6 \mathrm{~mL}, 56 \mathrm{mmol}\right)$ 
and TES $(19 \mathrm{~mL}, 112 \mathrm{mmol})$ were added to a $100 \mathrm{~mL}$ round bottom flask with a condenser and stirred at $24{ }^{\circ} \mathrm{C}$ for $5 \mathrm{~min}$, then TPFPB (57 mg, $0.112 \mathrm{~mol}$ ) was added into the mixture and reacted for another $3 \mathrm{~h}$. Subsequently, the mixture was passed through a neutral alumina column with dichloromethane as the eluent to eliminate TPFPB. Then the dichloromethane and unreacted TES in the collected mixture were removed by a vacuum rotary evaporator at $60{ }^{\circ} \mathrm{C}$. As-obtained eugenol protected by TES was present in a yellowish liquid. Finally, $5 \mathrm{~mL}$ of $0.1 \mathrm{M}$ hydrochloric acid aq. solution was added and stirred at $80{ }^{\circ} \mathrm{C}$ for $6 \mathrm{~h}$ to de-protection for obtaining $\mathrm{ABO}$. The chemical structure of TES protected eugenol and ABO were confirmed by ${ }^{1} \mathrm{H}$ NMR analysis (Section 1 of SI). The ABO grafted gauze (ABO-g-gauze) was made by the same way as that for USO-g-gauze, except that ABO was used to replace USO in the reaction mixture (Fig. 1b). HTMS with a long alkyl chain in its chemical structure was grafted onto the cotton gauze to make HTMS grafted gauze (HTMS-g-gauze) in a similar way for preparing USO-g-gauze (Fig. 1c).

\section{Characterization.}

The morphology of gauzes was observed by scanning electron microscopy (JEOL-7500LV, Japan). The solid-state ${ }^{13} \mathrm{C}$ NMR spectra were characterized by a superconducting fourier transform nuclear magnetic resonance spectrometer (Bruker Avance III $400 \mathrm{WB}$, AVANCE III, Switzerland). X-ray photoelectron spectra was measured by scanning XPS microprobe instrument (Thermo K-Alpha+, UK). Fourier transform infrared (FTIR) spectra in $\mathrm{KBr}$ form were obtained on an FTIR spectrometer (PerkinElmer, 1600) in the range of $4000-400 \mathrm{~cm}^{-1}$. The wettability was determined by DSA 100 (Krüss, Germany). 


\section{Moisture management test}

The tests (wetting time, wetted radius, water absorption rate, water spreading speed, and cumulative one-way transport capacity) were performed on a moisture management tester (M290, SDL ATLAS, USA) according to AATCC 195 by measuring the electrical resistance of the top and bottom sides of the gauze. ${ }^{33}$ The gauze size was $5 \mathrm{~cm} \times 5 \mathrm{~cm} .0 .2 \mathrm{~mL}$ of standard test solution was dropped onto the gauze in the test. 5 replicates were run for each gauze.

\section{Water vapor transmission rate and water absorption capacity of gauzes.}

To measure water vapor permeability, a beaker containing $50 \mathrm{~mL}$ of distilled water was covered with a gauze. The circumferential border was tightly sealed to prevent any water vapor loss through the boundary. The water vapor transmission rate $\left(\mathrm{W}_{\text {evap}}\right)$ was determined by measuring the mass loss of water in the beaker after $24 \mathrm{~h}$ at $37^{\circ} \mathrm{C}$. The $\mathrm{W}_{\text {evap }}\left(\mathrm{g} \cdot \mathrm{m}^{-2} \cdot \mathrm{day}^{-1}\right)$ was calculated according to Equation (1):

$$
\mathrm{W}_{\text {evap }}=\left(\mathrm{m}_{\mathrm{b}}-\mathrm{m}_{\mathrm{a}}\right) / \mathrm{A}
$$

where $A, m_{b}$, and $m_{a}$ are the area of the beaker mouth $\left(\mathrm{m}^{2}\right)$, the weight of the beaker before and after water evaporation, respectively.

For measurement of water absorption ratio, a square gauze with size of $2 \mathrm{~cm} \times 2$ $\mathrm{cm}$ was immersed in simulated body fluid (SBF), then at certain time interval it was taken out and placed on a filter paper to absorb free water, followed by weighing its mass. The gauze mass before $\left(\mathrm{m}_{\mathrm{b}}\right)$ and after water absorption $\left(\mathrm{m}_{\mathrm{a}}\right)$ was measured, and water absorption ratio $\left(\mathrm{W}_{\mathrm{abs}}\right)$ was calculated by Equation (2):

$$
\mathrm{W}_{\mathrm{abs}}=\left(\mathrm{m}_{\mathrm{a}}-\mathrm{m}_{\mathrm{b}}\right) / \mathrm{m}_{\mathrm{b}} \times 100 \%
$$




\section{Hemostasis evaluation}

All animal experiments were carried out in accordance with the guidelines for the protection and use of experimental animals in Fujian Normal University. The experiments were approved by the Animal Ethics Committee of Fujian Normal University. The gauze was tailored into rectangle swatch with a size of $12 \mathrm{~cm} \times 2.5 \mathrm{~cm}$, and four swatches were stacked together before used on a bleeding wound. The hemostatic study included four animal injury models: rat femoral artery and liver injury, pig femoral artery and skin laceration. Five rats or pigs were randomly selected as a group in each animal model and assigned to each sample. QCG is the standard military hemostatic agent recommended by the Tactical Combat Injury Care Committee for use as a control. Anesthesia was injected intraperitoneally with a $10 \mathrm{wt} \%$ chloral hydrate solution $(0.4 \mathrm{~mL} / 100 \mathrm{~g})$. After complete hemostasis was reached, all animals were observed for $2 \mathrm{~h}$ or until death. The survived animals were euthanized with $10 \%$ chloral hydrate at the end of experiment.

\section{Rat femoral artery injury model}

The proceeding of femoral artery injury model was conducted as follows. ${ }^{34}$ Rats were randomly selected and anesthetized. Then the fur on the rat thigh was shaved off to expose the femoral artery. Pre-weighed cotton gauze was placed beneath the thigh. Then the artery was transected. After bleeding for $2 \mathrm{~s}$, pre-weighed gauze was gently applied or compressed onto the trauma (compressing for $150 \mathrm{~s}$ ). Blood diffusion in the gauze was recorded. Bleeding time and blood loss were measured $(n=6)$. We defined that hemostatic stage is reached when the blood-stained area on gauze doesn't expand and no blood seeps out of the seam at gauze/wound contact surface.

For observing the micro-morphology of the blood-stained gauze after hemostasis, a patch stacked with four gauze layers was dressed onto the rat femoral artery injury to reach 
1 hemostasis. The gauze was placed in centrifuge tubes and fixed with $2.5 \%(\mathrm{v} / \mathrm{v} \%) \mathrm{GA} / \mathrm{PBS}$

2 solution at room temperature for $4 \mathrm{~h}$. Then it was rinsed twice with distilled water, followed

3 by sequential dehydration in $0 \%, 10 \%, 20 \%, 30 \%, 40 \%, 50 \%, 60 \%, 70 \%, 80 \%, 90 \%$ and

$4 \quad 100 \%(\mathrm{v} / \mathrm{v} \%)$ ethanol/PBS solution. consequently, it was dried at $37{ }^{\circ} \mathrm{C}$. The gauze was

5 sputter-coated with gold before SEM observation.

6

$7 \quad$ Rat liver laceration model

8 $14=6)$.

Liver laceration model was conducted according to a standard procedure. ${ }^{35}$ Rats were randomly selected and anesthetized. Subsequently, the epithelial tissue of the abdomen was cut to expose the liver, and pre-weighted cotton gauze were placed under the liver. A scalpel was used to make a linear incision trauma of about $1 \mathrm{~cm}$ in length on the left lobe of the liver. After bleeding for $2 \mathrm{~s}$, pre-weighed gauze was applied onto the trauma. Blood permeation in the gauze, bleeding time, and blood loss were recorded (n

\section{Pig skin laceration and femoral artery injury models}

Two-month-old Bama miniature pigs (weight 1.6 - $1.8 \mathrm{~kg}$ ) were used to simulate massive bleeding trauma. ${ }^{36}$ Anesthesia was injected intraperitoneally with a $10 \mathrm{wt} \%$ chloral hydrate solution $(0.4 \mathrm{~mL} / 100 \mathrm{~g})$. After the hairs on the back and leg were shaved off, the following two different traumas were created: (1) A linear incision of $2 \mathrm{~cm}$ (length) $\times 1 \mathrm{~cm}$ (depth) was made on the back of the pig with a scalpel; (2) A distally extending $4 \mathrm{~cm}$ longitudinal incision was made in the right femur region to expose the femoral artery. ${ }^{37}$ 
Peeling force of gauze on wet rat femoral tissue

Rats were pre-treated as in the femoral artery injury model, then the peeling test was conducted before the artery was transected. Gauze $\left(5 \times 2.5 \mathrm{~cm}^{2}\right)$ was immediately put on the exposed wet femoral tissue and kept there for $10 \mathrm{~min}$. The gauze was then peeled off with a Digital Push Pull Gauge (Locosc Ningbo Precision Technology Co., Ltd., China), and the peak force was recorded $(n=5)$.

\section{Effect of catechol functional groups on hemostasis}

In order to elucidate the effect of catechol structure on hemostatic efficiency of USO-g-gauze, two models were designed: (1) The catechol groups were protected by chelating with $\mathrm{Fe}^{3+}$ ions. ${ }^{38}$ Typically, USO-g-gauze was soaked in $100 \mathrm{~mL}$ of $0.1 \mathrm{M} \mathrm{FeCl}_{3}$ aq. solution for 10 minutes at $37{ }^{\circ} \mathrm{C}$ to allow the occurrence of coordination reaction between catechol groups and $\mathrm{Fe}^{3+}$. After washed with distilled water, it was dried in a vacuum oven at $80{ }^{\circ} \mathrm{C}$ for $0.5 \mathrm{~h}$. As-obtained gauze was coded as USOFe-g-gauze. (2) The catechol groups were oxidized to quinone. The USO-g-gauze was treated in $100 \mathrm{~mL}$ of $20 \mathrm{mM}$ Tris- $\mathrm{HCl}$ buffer solution ( $\mathrm{pH} 9.8)$ for 10 minutes at $37{ }^{\circ} \mathrm{C}$ to convert the phenolic structure to quinoine structure. ${ }^{39}$ After washed with distilled water, it was dried in a vacuum oven at $80{ }^{\circ} \mathrm{C}$ for $0.5 \mathrm{~h}$. As-obtained gauzed was coded as USOQu-ggauze.

\section{Accumulation of erythrocytes and platelets on gauzes}

The platelet-rich plasma (PRP) was obtained by centrifuging rat whole blood at $3000 \mathrm{rpm}$ for $20 \mathrm{~min}$ at $4{ }^{\circ} \mathrm{C} .{ }^{40} \mathrm{In}$ a volume ratio of $1: 10$, the whole blood or PRP was 
1 added into a phosphate buffer solution (PBS, $\mathrm{pH} 7.4)$ containing a piece of gauze $(1 \mathrm{~cm}$

$2 \times 1 \mathrm{~cm}$ ), followed by incubating for $90 \mathrm{~min}$ at $37^{\circ} \mathrm{C}$. Subsequently, the gauze was rinsed

3 three times with PBS to remove physically adhered blood cells and platelets, and fixed

4 with $2.5 \%(\mathrm{v} / \mathrm{v} \%) \mathrm{GA} / \mathrm{PBS}$ for $2 \mathrm{~h}$. Then it was rinsed twice with distilled water,

5 followed by sequentially dehydrating with $25 \%, 50 \%, 75 \%, 85 \%, 90 \%$, and $100 \%$ (v/v\%)

6 ethanol/PBS solution. Finally, it was air-dried at $37^{\circ} \mathrm{C}$. The adhesion of erythrocyte and

7 platelet on gauze surface was observed by SEM.

9 Biocompatibility of gauze

The proliferation of L929 cells on gauze was measured by using a live/dead assay

11 kit (Beyotime Biotechnology, Shanghai, China). ${ }^{41}$ Briefly, after treated with $75 \%$ medical alcohol and $0.1 \mathrm{mg} / \mathrm{mL}$ physiological saline, the gauze was transferred into a 24-well plastic culture plate. $1 \mathrm{~mL}$ of fibroblast with a density of $2.5 \times 10^{5}$ cells $/ \mathrm{mL}$ was gently added on the surface of the gauze surface and cultured at $37^{\circ} \mathrm{C}$ in a humidified atmosphere of $5 \% \mathrm{CO}_{2}$ for 1,2 , and $3 \mathrm{~d}$. Then, $10 \mu \mathrm{L}$ of the combined Live/Dead cell-

16 staining solution ( $2 \mu \mathrm{M}$ Calcein-AM and $4 \mu \mathrm{M}$ PI) was added into $400 \mu \mathrm{L}$ of culture medium and was incubated at $37^{\circ} \mathrm{C}$ for $4 \mathrm{~h}$. Finally, images of the live (green fluorescence) and dead (red fluorescence) cells were obtained using an inverted

19 fluorescence microscope.

In vivo inflammatory assay 
1 divided into 2 groups: cotton gauze group and USO-g-gauze. Each group contained 20

2 rats. The gauze was cut into a square $(1 \mathrm{~cm} \times 1 \mathrm{~cm})$ and were sterilized by ultraviolet

3 irradiation for $2 \mathrm{~h}$. The rats were anesthetized by inhalant anesthetics-ether and fixed on

4 the surgical plate. After the shave and the iodine disinfection, a longitudinal incision

5 about $2 \mathrm{~cm}$ were made symmetrically on both sides of the spine. Then, the cotton gauze

6 or USO-g-gauze were respectively implanted into the subcutaneous sac of the back. The

7 incision was sutured by the simple intermittent suture method. Finally, the cut was

8 sprayed with penicillin powder and then covered with aseptic dressing paste to prevent

9 infection. The animals were returned to cages alone after surgery. At specific times (3,

$107,14,21$ days), the rats were sacrificed and the wounds along with surrounding tissues

11 were collected. The collected tissues were fixed by $4 \%$ paraformaldehyde. After

12 formaldehyde fixation for 1 week, the calcium was decalcified with $10 \%$ EDTA reagent

13 for 2-4 weeks. Subsequently, H\&E staining and toluidine blue staining were performed,

14 and staining was performed using a fluorescence inverted microscope for microscopic

15 examination and image acquisition analysis.

\section{Statistical analysis}

All data are shown as the mean \pm standard deviation (SD) with five replicates for each test. One-way analysis of variance (ANOVA) was applied for statistical analysis. ${ }^{*} \mathrm{p}<0.05$ and $* * \mathrm{p}<0.01$ were considered significant and greatly significant difference, respectively. 


\section{Acknowledgements}

This work is supported by Natural Science Foundation of China (22175037, 52103108, 21673093 and U1805234), Social Development of Instructive Program of Fujian Province (2020Y0020), Natural Science Foundation of Fujian Province (2020J01147), Minjiang Scholar and startup fund for high-level talent at Fujian Normal University for Prof. W. Chen. We thank Prof. Ran Li of Fujian Agriculture and Forestry University, and Prof. Lixin Wu of Fujian Institute of Research on the Structure of Matter for plasma treatment of cotton gauze, Dr. Kunkun Zhu of Wuhan Textile University for moisture management test.

\section{Author contributions}

H. Liu and Y. Fang conceived and directed the project. H. He, W. Zhou, A. Liu, W. Zhang, and Y. Weng designed and performed the synthesis, characterization and hemostatic measurements of gauzes. F. Wang, S. Wang and Z. Wang carried out the tests of biocompatibility. J. Gao, Y. Gao and W. Chen run the density functional theory calculation and analysis. H. Liu., and W. Chen wrote the manuscript.

\section{Competing interests}

The authors declare no competing interests.

\section{Reference}

1. Kauvar, D.S., Lefering, R. \& Wade, C.E. Impact of Hemorrhage on Trauma Outcome: An Overview of Epidemiology, Clinical Presentations, and Therapeutic Considerations. J. Trauma 60, S3-11 (2006).

2. $\mathrm{Du}, \mathrm{X}$. et al. Microchannelled alkylated chitosan sponge to treat noncompressible hemorrhages and facilitate wound healing. Nat. Commun. 12, 4733 (2021).

3. Hsu, B.B. et al. Clotting mimicry from robust hemostatic bandages based on selfassembling peptides. ACS Nano 9, 9394 (2015).

4. Fang, Y. et al. 3D porous chitin sponge with high absorbency, rapid shape 
recovery, and excellent antibacterial activities for noncompressible wound. Chem. Eng. J. 388, 124169 (2020).

5. Hickman, D., Pawlowski, C., Sekhon, U.D., Marks, J. \& Gupta, A. Biomaterials and advanced technologies for hemostatic management of bleeding. Adv. Mater. 30, 1700859 (2018).

6. Gao, Y. et al. A polymer-based systemic hemostatic agent. Sci. Adv. 6, eaba0588 (2020).

7. Wang, Y.M. et al. Chitosan-bound carboxymethylated cotton fabric and its application as wound dressing. Carbohydr. Polym. 221, 202-208 (2019).

8. $\quad \mathrm{Yu}, \mathrm{L} . \mathrm{S}$. et al. A tightly-bonded and flexible mesoporous zeolite-cotton hybrid hemostat. Nat. Commun. 10, 1932 (2019).

9. Leonhardt, E.E., Kang, N., Hamad, M.A., Wooley, K.L. \& Elsabahy, M. Absorbable hemostatic hydrogels comprising composites of sacrificial templates and honeycomb-like nanofibrous mats of chitosan. Nat. Commun. 10, 2307 (2019).

10. Shin, M. et al. Complete prevention of blood loss with self-sealing haemostatic needles. Nat. Mater. 16, 147 (2017).

11. Yuk, H. et al. Dry double-sided tape for adhesion of wet tissues and devices. Nature 575, 169 (2019).

12. Li, J. et al. Tough adhesives for diverse wet surfaces. Science 357, 378 (2017).

13. Zhu, T. et al. Superhydrophobic/superhydrophilic janus fabrics reducing blood loss. Adv. Healthcare Mater. 7, 1701086 (2018).

14. Li, Z. et al. Superhydrophobic hemostatic nanofiber composites for fast clotting and minimal adhesion. Nat. Commun. 10, 5562 (2019).

15. Spotnitz, W.D. \& Burks, S. Hemostats, sealants, and adhesives: components of the surgical toolbox. Transfusion 48, 1502 (2008).

16. Wang, R. et al. A biomimetic mussel-inspired epsilon-poly-L-lysine hydrogel with robust tissue-anchor and anti-infection capacity. Adv. Funct. Mater. 27, 1604894 (2017).

17. Cui, C. et al. Water-triggered hyperbranched polymer universal adhesives: From strong underwater adhesion to rapid sealing hemostasis. Adv. Mater. 31, 1905761 (2019).

18. Tokoh, C., Takabe, K., Sugiyama, J. \& Fujita, M. CP/MAS ${ }^{13} \mathrm{C}$ NMR and electron diffraction study of bacterial cellulose structure affected by cell wall polysaccharides. Cellulose 9, 351-360 (2002).

19. Lao, L., Shou, D., Wu, Y.S. \& Fan, J.T. "Skin-like" fabric for personal moisture management. Sci. Adv. 6, eaaz0013 (2020).

20. Švorčík, V. et al. Modification of surface properties of high and low density polyethylene by Ar plasma discharge. Polym. Degrad. Stabil. 91, 1219-1225 (2006).

21. Díez-Pascual, A. \& Díez-Vicente, A. Wound healing bionanocomposites based on castor oil polymeric films reinforced with chitosan-modified zno nanoparticles. Biomacromolecules 16, 2631-2644 (2015).

22. Su, C., Zhao, H., Yang, H. \& Chen, R. Stearic acid-modified starch/chitosan composite sponge with asymmetric and gradient wettability for wound dressing. ACS Appl. Bio Mater. 2, 171-181 (2019).

23. Wen, J., Weinhart, M., Lai, B., Kizhakkedathu, J. \& Brooks, D.E. Reversible hemostatic properties of sulfabetaine/quaternary ammonium modified hyperbranched polyglycerol. Biomaterials 86, 42-55 (2016).

24. Sekhon, U.D. \& Gupta, A. Platelets and Platelet-Inspired Biomaterials Technologies in Wound Healing Applications. ACS Biomater. Sci. Eng. 4, 1176- 
1192 (2017).

25. Fuchs, E. Skin stem cells: rising to the surface. J. Cell Biol. 180, 273-284 (2008).

26. Xie, $L$. et al. A wet adhesion strategy via synergistic cation- $\pi$ and hydrogen bonding interactions of antifouling zwitterions and mussel-inspired binding moieties. J. Mater. Chem. A 7, 21944 (2019).

27. Kaur, S. et al. Direct observation of the interplay of catechol binding and polymer hydrophobicity in a mussel-inspired elastomeric adhesive. ACS Central Sci. 4, 1420 (2018).

28. Fan, X. et al. Mussel foot protein inspired tough tissue-selective underwater adhesive hydrogel. Mater. Horizon 8, 997-1007 (2021).

29. Saiz-Poseu, J., Mancebo-Aracil, J., Nador, F., Busqué, F. \& Ruiz-Molina, D. The chemistry behind catechol-based adhesion. Angew. Chem. Int. Ed. 58, 696 (2019).

30. Fan, X. et al. An anti-freezing/anti-heating hydrogel containing catechol derivative urushiol for strong wet adhesion to various substrates. ACS Appl. Mater. Interface 12, 32031-32040 (2020).

31. Kumanotani, J. Urushi (oriental lacquer) - a natural aesthetic durable and futurepromising coating. Prog. Org. Coat. 26, 163-195 (1995).

32. Ahn, B.K., Lee, D.W., Israelachvili, J.N. \& Waite, J.H. Surface-initiated selfhealing of polymers in aqueous media. Nat. Mater. 13, 867-872 (2014).

33. Chen, Q. et al. The Moisture Management and Drying Properties of Weft Knitted Plating Fabrics. Fiber Polym. 21, 1347-1354 (2020).

34. Wang, C. et al. Tannic acid-loaded mesoporous silica for rapid hemostasis and antibacterial activity. Biomater. Sci. 6, 3318-3331 (2018).

35. Chen, Z. et al. A rapid hemostatic sponge based on large, mesoporous silica nanoparticles and N-alkylated chitosan. Nanoscale 10, 20234-20245 (2018).

36. Bu, Y.Z. et al. Synthesis and properties of hemostatic and bacteria-responsive in situ hydrogels for emergency treatment in critical situations. ACS Appl. Mater. Interface 8, 12674 (2016).

37. White, N.J. et al. Rediscovering the wound hematoma as a site of hemostasis during major arterial hemorrhage. J. Thromb. Haemost. 13, 2202-2209 (2015).

38. Guo, Z., Ni, K., Wei, D. \& Ren, Y. Fe ${ }^{3+}$-induced oxidation and coordination crosslinking in catechol-chitosan hydrogels under acidic pH conditions. RSC Adv. 5, 37377-37384 (2015).

39. Das, P. \& Reches, M. Revealing the role of catechol moieties in the interactions between peptides and inorganic surfaces. Nanoscale 8, 15309-15316 (2016).

40. Ong, S.Y., Wu, J., Moochhala, S.M., Tan, M.H. \& Lu, J. Development of a chitosan-based wound dressing with improved hemostatic and antimicrobial properties. Biomaterials 29, 4323-4332 (2008).

41. Gao, H. et al. Construction of cellulose nanofibers/quaternized chitin/organic rectorite composites and their application as wound dressing materials. Biomater. Sci. 7, 2571-2581 (2019). 


\section{Supplementary Files}

This is a list of supplementary files associated with this preprint. Click to download.

- MSsubmittedtoNC20210831SPI.pdf

- SupplementaryMovie1.mp4

- SupplementaryMovie2.mp4

- SupplementaryMovie3.mp4

- SupplementaryMovie4.mp4

- SupplementaryMovie5.mp4

- SupplementaryMovie6.mp4

- SupplementaryMovie7.mp4 